\title{
ANALYSE DES STATISTIQUES DE PÊCHE AUX ENGINS DANS LE BASSIN DU RHÔNE.
}

\section{PREMIÈRE PARTIE : ÉTUDE DE L'EFFORT DE PÊCHE.}

\section{T. CHANGEUX (1) et M. ZYLBERBLAT (2)}

(1) Laboratoire d'Ecologie des Systèmes Fluviaux, 1 rue Parmentier, 13200 ARLES, France.

(2) Direction Régionale de l'Environnement Rhône-Alpes, 12 Quai Maréchal Joffre, 69002 LYON, France.

\section{RÉSUMÉ}

La majorité du linéaire constitué par les parties navigables du Rhône et de la Saône est exploitée par la pêche professionnelle fluviale ou amateur aux filets et engins. II n'existe pas d'autorisation administrative permettant de distinguer précisément les différents métiers de pêche qui sont à définir.

Durant les années 1988 et 1989 , le nombre de pêcheurs a été estimé à 37 professionnels et 708 amateurs. Ils se concentrent particulièrement en Saône aval et accessoirement dans le Rhône amont et le Rhône deltaîque.

Pendant ces mêmes années, une déclaration volontaire des captures et de l'effort de pêche a été expérimentée sur l'ensemble de la pêcherie. L'utilisation des statistiques administratives et des réponses, obtenues avec un taux de $47 \%$, permettent d'extrapoler nos résultats à l'ensemble de la pêcherie.

L'activitè de pêche suit un rythme saisonnier : après une fermeture partielle, en mai, la plus forte affluence s'établit à partir de juin et jusqu'en novembre. La pêcherie est dominée par l'usage des pièges fixes chez les professionnels et des carrelets chez les amateurs. On peut distinguer 3 métiers chez les pêcheurs professionnels et 5 métiers chez les pêcheurs amateurs. L'effort de pêche se diversifie particulièrement en juin, juillet, août avec l'arrivée des amateurs.

Le nombre important d'amateurs permet de meilleures estimations du nombre d'engins que pour les professionnels. Les résultats de l'analyse des captures feront l'objet de la seconde partie qui suit.

Mots-clés : pêcherie, engins, enquête, effort de pêche, bassin du Rhône.

\section{ANALYSIS OF FISHING GEAR FISHERY STATISTICS IN THE RHÔNE RIVER BASIN.}

PART ONE : STUDY OF THE FISHING EFFORT.

\begin{abstract}
The majority of the navigable waters of the Rhône and the Saône rivers is exploited by fishermen, either commercial fishermen (professionals) or recreational fishing gear users (amateurs). Besides these two categories, no further distinctions are clearly defined by the administrative authorities, and more precise characterizations are needed to standardize fishing effort.
\end{abstract}


During the years 1988 and 1989, the number of fishermen were estimated at 37 professionals and 708 amateurs. They were concentrated primarily in the lower Saône and secondarily in the upper Rhône and the deltaic Rhône.

In this same period, we experimented with a voluntary declaration of capture and fishing effort over the whole fishery. The use of these responses, obtained at a $47 \%$ answer rate, in conjunction with the administrative statistics allowed us to extrapolate our results to the whole fishery.

Fishing activity follows a seasonal pattern : after a partial closing in May, the main effort establishes itself in June and lasts until November. The fishery is dominated by the use of fish traps by professionals and lift nets by amateurs. We can distinguish 3 categories of fishing for the professionals, and 5 for the amateurs. The fishing effort becomes especially diversified in June, July, and August, following the arrival of amateurs.

The large number of amateurs results in a better estimate of the number of fishing gear than it is possible with professionals. The results of the capture analysis are the subject of the second part which follows.

Key-words : fishery, fishing gear, survey, fishing effort, Rhône River basin.

\section{INTRODUCTION}

Faute d'estimations fiables, les productions mondiales des eaux continentales sont, à l'heure actuelle, sous-évaluées (WELCOMME, 1985). Elles sont estimées à $14 \%$ du tonnage mondial des apports par pêche (FAO, 1992, p. 85). Comptant seulement pour $3,5 \%$ de cette production, les eaux douces européennes ont perdu l'essentiel de leur rôle alimentaire devant la concurrence des produits marins.

En France, la pêche aux engins rassemble deux activités fondamentalement différentes : une pêche de loisir dite amateur, dont les captures sont légalement interdites à la vente, et une pêche commerciale dite professionnelle. Avec 45000 tonnes en 1989, les productions commerciales françaises de poissons d'eau douce et diadromes déclarées sont marquées par l'importance de la pisciculture (31 000 tonnes de truites arc-en-ciel de pisciculture (Oncorhynchus mykiss Walbaum 1792), soit le premier tonnage mondial pour cette espèce (FAO, 1992)). Le reste est essentiellement issu des étangs, soit : 8000 à 15000 tonnes/an (ARRIGNON, 1991 ; ANONYME, 1992a). Les productions de la pêche professionelle continentale, marins pêcheurs exclus, sont évaluées au maximum à 2000 tonnes (ANONYME, 1992a ; BABIN, 1991). Cette activité concerne plus de 1200 pêcheurs pour un chiffre d'affaires de 80 millions de francs départ pêcheur (CASTELNAUD et BABIN, 1992). En revanche, on dénombre 1,9 millions de pêcheurs à la ligne déclarés pour une dépense globale associée évaluée à 6 milliards de francs (ANONYME, 1992b). Pour WELCOMME (1978, in DILL, 1990), la gestion piscicole des eaux douces est actuellement, dans la majorité des pays industrialisés, un enjeu économique lié en premier lieu au tourisme, à la pêche de loisir, et à la pisciculture.

Depuis longtemps déjà, les gestionnaires déplorent l'absence de statistiques de pêche fiables pour un suivi scientifique et économique des grands cours d'eau français (PERAU, 1930 ; CECPI, 1978). Cet article, séparé en deux parties, évalue, sur les deux années de référence 1988 et 1989, l'utilisation des déclarations de captures proposée par le cahier des charges (annexe du décret du 28 août 1987) pour le suivi à long terme du bassin du Rhône. II s'inscrit en complément des suivis halieutiques des estuaires de la façade atlantique entamés en 1978 (CTGREF, 1979, in ELIE et al., 1989) et des études effectuées à l'échelon national concernant la pêche commerciale (CASTELNAUD et BABIN, 1992 ; BABIN, 1991 ; CASTELNAUD et al., 1985). Dans ce travail nous avons ajouté aux informations fournies par les pêcheurs professionnels celles, inédites, des pêcheurs amateurs. Limité au bassin du Rhône, notre étude répond à un objectif biologique qui concerne davantage les ressources piscicoles que les pêcheurs eux-mêmes. Car, en dehors de leur intérêt socio-économique, leurs déclarations peuvent être utilisées comme outils de gestion pour orienter les modalités d'exploitation de la ressource dans le cadre d'une surveillance écologique des milieux aquatiques telle qu'elle est inscrite dans la loi sur la qualité des eaux de 1964 et la loi sur la pêche en eau douce de 1984 . 
Dans cette première partie à vocation méthodologique, nous effectuerons tout d'abord une rapide présentation de l'hydrologie et de la morphologie du bassin du Rhône définissant le milieu d'application de l'étude. Nous exposerons, ensuite, un protocole permettant d'obtenir l'estimation chiffrée de l'effort de pêche et des captures déclarées ainsi que leurs domaines de confiance en associant les statistiques administratives et les déclarations des pêcheurs. Puis, une analyse approfondie de l'effort de pêche dégagera les métiers de pêche amateur et professionnelle qui seront utilisés dans la seconde partie de l'article consacrée à l'estimation des captures et à leur utilisation pour fournir un indice d'abondance des stocks exploités.

\section{PRÉSENTATION DU MILIEU}

Avec une surface de $95000 \mathrm{~km}^{2}$, dont $93 \%$ se trouvent en France, le bassin du Rhône couvre $15 \%$ du territoire national. En dehors des lacs alpins et des étangs privés, la pêche aux engins n'y est admise que dans le Domaine Public Fluvial (DPF). La généralisation de nos statistiques à la totalité du DPF du bassin (plus de $2200 \mathrm{~km}$ de linéaire) ne s'est faite que progressivement, la priorité étant donnée au fleuve Rhône et à la Saône où se concentrent l'essentiel des pêcheurs aux engins. Cinq secteurs d'exploitation, correspondant aux grands ensembles géographiques du réseau, ont été distingués pour localiser les résultats (Fig. 1 et Tableau I).

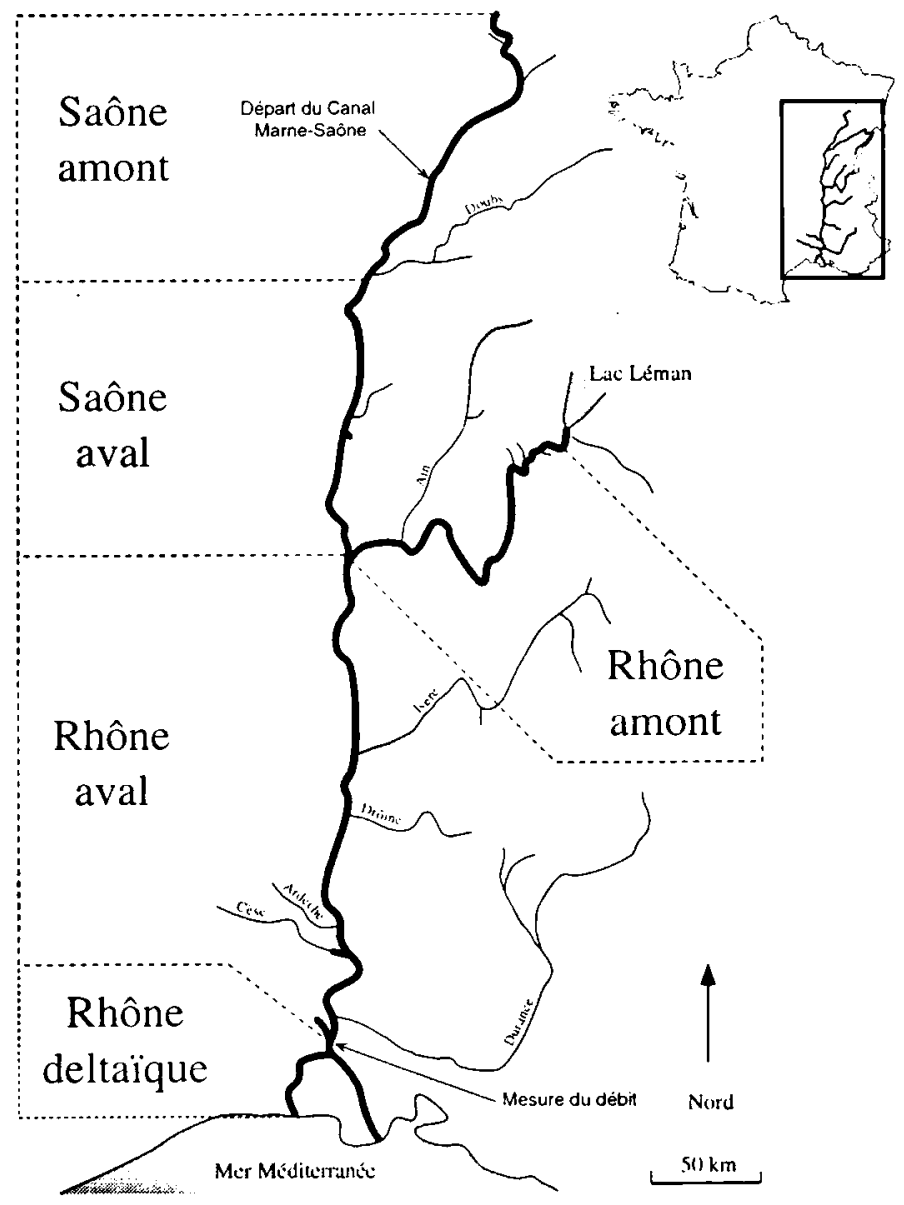

Figure 1 : Le Domaine Public Fluvial du bassin du Rhône montrant les cinq secteurs géographiques d'exploitation.

Figure 1: State River Property in the Rhône watershed showing the five sectors of exploitation. 
Ce réseau est subdivisé en tronçons de 0,2 à $25 \mathrm{~km}$ de long, appelés lots, dont un nombre limité est ouvert à la pêche aux engins par autorisation administrative. Les autorisations sont délivrées individuellement pour chaque lot de pêche. Les lots ont été décrits sur la base des travaux déjà effectués sur le Rhône (GALLOIS, 1947 ; PERSAT, 1988 ; MALAVOI, 1989 ; FRUGET, 1989) en distinguant des unités d'environnement homogènes sur $200 \mathrm{~m}$ de longueur de rive au minimum. L'extension de ce procédé à la Saône, uniquement en aval du canal Marne-Saône, conduit à différencier 7 milieux types :

- bras principal : section par où transite l'essentiel du débit ;

- bras secondaire : annexe latérale du cours d'eau par où transite une minorité du débit ;

- plan d'eau : annexe isolée du cours d'eau, mise en eau uniquement lors des plus grandes crues annuelles ;

- affluent : confluence d'un affluent incluse dans le DPF ;

— canal : tronçon latéral dont les rives sont bétonnées et très pentues ;

- retenue: portion d'un ouvrage hydroélectrique de type basse chute située en amont du barrage et consacrée au stockage de l'eau ;

- lit court-circuité : ancien lit du cours d'eau situé en aval du barrage soumis au débit réservé.

Les deux premiers milieux se rapportent à des portions de cours d'eau dont les aménagements structurants datent du siècle dernier. Les deux derniers sont strictement attachés aux modifications postérieures à 1952.

Tableau I : Structure d'exploitation (nombre de lots, tailles moyennes) et distribution des milieux types (en linéaire et en abondances relatives) du domaine d'étude suivant les cinq secteurs géographiques retenus pour la présentation des résultats. Les intervalles de confiance sont indiqués au risque alpha de $5 \%$.

Table I : Structure of the fishery exploitation (number of lot, average length) and distribution of the environmental types (length and relative abundance) in the study area following the five exploitation sectors where fishery statistics are grouped. The confidence intervals are given at an alpha value of $5 \%$.

\begin{tabular}{|c|c|c|c|c|c|c|c|c|c|c|c|c|}
\hline \multirow{2}{*}{$\begin{array}{c}\text { SECTEUR } \\
\text { D'EXPLOITATION } \\
\text { Nombre de lots } \\
\text { Taile moyenne }(\mathrm{km}) \\
\end{array}$} & \multicolumn{2}{|c|}{ RHÔNE AMONT } & \multicolumn{2}{|c|}{ RHÔNE AVAL } & \multicolumn{2}{|c|}{$\begin{array}{c}\text { RHÔNE } \\
\text { DELTÄ̈QUE }\end{array}$} & \multicolumn{2}{|c|}{ SAÔNE AMONT } & \multicolumn{2}{|c|}{ SAÓNE AVAL } & \multicolumn{2}{|c|}{ TOTAL. } \\
\hline & $\begin{array}{r}41 \\
5,3( \pm\end{array}$ & $0,5)$ & 5,8 & $0,8)$ & 16,5 & $+4,4)$ & $\begin{array}{r}13 \\
2,1 \pm\end{array}$ & $\begin{array}{l}5 \\
0,1)\end{array}$ & 3,0 & $\begin{array}{l}8 \\
0,4)\end{array}$ & $\begin{array}{r}29 \\
3.7 \pm\end{array}$ & $0,3)$ \\
\hline MILIEU TYPE & $\begin{array}{c}\text { Linéaire } \\
(\mathrm{km})\end{array}$ & $\%$ & $\begin{array}{l}\text { Linéaire } \\
(\mathrm{km})\end{array}$ & $\%$ & $\begin{array}{l}\text { Linéaire } \\
(\mathrm{km})\end{array}$ & $\%$ & $\begin{array}{l}\text { Linéaire } \\
(\mathrm{km})\end{array}$ & $\%$ & $\begin{array}{c}\text { Linéaire } \\
(\mathrm{km})\end{array}$ & $\%$ & $\begin{array}{l}\text { Linéaire } \\
(\mathrm{km})\end{array}$ & $\%{ }^{*}$ \\
\hline Bras principal & 51,9 & 24,0 & 35,3 & 11,2 & 96,5 & 83,5 & 91,1 & 80,3 & 147,7 & 86,0 & 422,5 & 45,4 \\
\hline Bras secondaire & 5,3 & 2,5 & 15,2 & 4,8 & & & 5,1 & 4,5 & 14,8 & 8,6 & 40,4 & 4,3 \\
\hline Plan d'eau & & & 8,0 & 2,5 & & & 4,8 & 4,2 & 0.7 & 0,4 & 13,5 & 1,4 \\
\hline Affluent & & & 10,7 & 3,4 & & & & & 5,1 & 3,0 & 15,8 & 1,7 \\
\hline Canal & 48,6 & 22,5 & 44,3 & 14,1 & 3,8 & 3,3 & 12,5 & 11.0 & 3,5 & 2,0 & 112,6 & 12,1 \\
\hline Retenue & 75,2 & 34,8 & 117,1 & 37,3 & 9,5 & 8,2 & & & & & 201,8 & 21,7 \\
\hline Lit court-circuité & 35,2 & 16,3 & 83,8 & 26,7 & 5,7 & 4,9 & & & & & 124,7 & 13,4 \\
\hline Non décrit & & & & & & & 174,3 & & & & 174,3 & \\
\hline Total & 216,2 & 100,0 & 314,3 & 100,0 & 115,5 & 100,0 & 287,8 & 100,0 & 171,8 & 100,0 & 1105,5 & 100,0 \\
\hline
\end{tabular}

* Linéaire non décrit exclu. 
La distribution des milieux (Tableau I) concorde avec les résultats obtenus par COTTEREAU (1988) sur le Rhône uniquement, sachant que notre définition des milieux types chevauche la classification utilisée par cet auteur. Les secteurs de la Saône et du Rhône deltaïque n'ont pas été récemment modifiés. Ils se distinguent clairement du Rhône amont et du Rhône aval par un linéaire constitué à plus de $80 \%$ par un milieu de type "bras principal". Entre la Saône aval et la Saône amont, le milieu de type "canal" progresse de 2 à $11 \%$ car la mise en place de ces ouvrages est rendue nécessaire pour la navigation au fur et à mesure que l'on remonte une rivière.

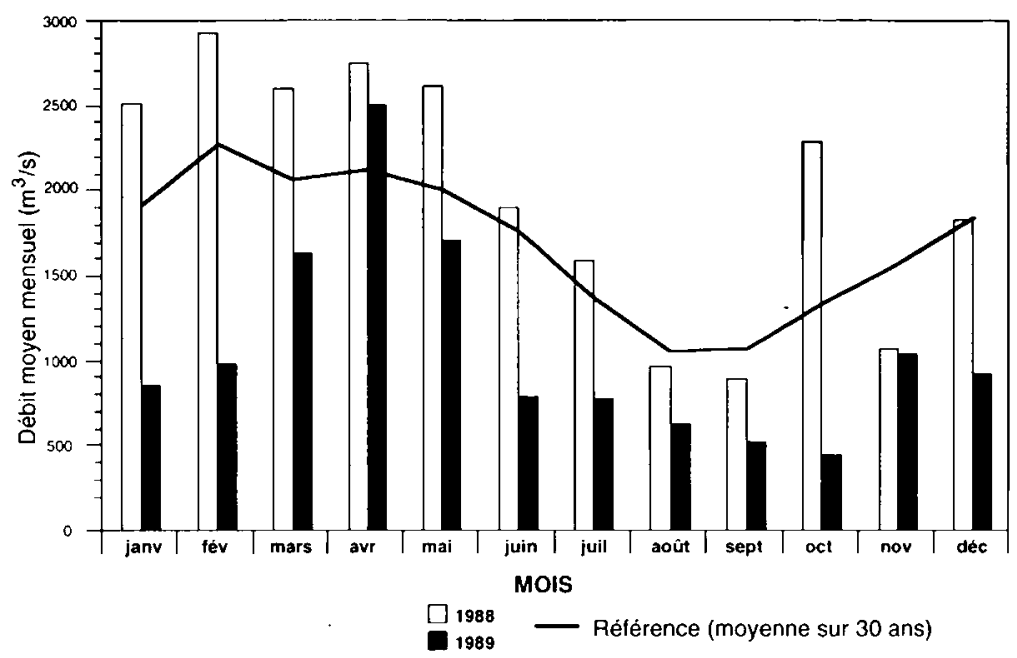

Figure 2 : Débits moyens mensuels du Rhône au sommet du delta (Baucaire). Référence et valeurs pour 1988 et 1989 mettant en évidence le contraste hydrologique entre les deux années. (source : C.N.R.)

Figure 2 : Average monthly discharge of the Rhône River at the head of the delta (Baucaire). The values given for 1988 and 1989 show the contrast between two hydrologically different years. The $\mathbf{3 0}$-year average is given for reference. (source : C.N.R.)

Le régime hydrologique du Rhône, à proximité de son émissaire, est de type pluvial tempéré avec de fortes eaux en hiver et un étiage en fin d'été (Fig. 2). Les deux années couvertes par nos statistiques apparaissent de ce point de vue très contrastées. Le module 1988 est légèrement au dessus du module de référence alors que celui de 1989 n'en représente que $62 \%$. L'importance du module de la première année relève du dépassement continuel du débit de référence mensuel entre janvier et juillet ajouté à une crue importante au mois d'octobre. En revanche, les débits 1989 se situent généralement en dessous du débit de référence avec un étiage particulièrement sévère au mois d'octobre.

La proportion de l'espace étudié, considérée comme exploitable par la pêche aux engins selon l'administration, s'élève à $73,9 \%$ du linéaire (Tableau II). Les diminutions de fréquences des milieux types entre domaine étudié et domaine exploitable s'interprètent de deux manières différentes. D'une part, l'administration est censée limiter son offre aux pêcheurs aux engins dans les secteurs où cette pêche risque d'entraîner un déséquilibre biologique. II s'agit généralement des sites où intervient la concurrence des pêcheurs à la ligne ("Lit court-circuité", "Bras secondaire" et "Plan d'eau"). II suffit qu'un lot soit momentanément inoccupé par les pêcheurs aux engins pour que les pêcheurs à la ligne obtiennent sa mise en réserve définitive. D'autre part, l'administration adapte son offre à la demande sur des secteurs pauvres et difficilement pêchables ("Canal") ou sujets à déprise ("Non décrit" de la Saône amont).

Sur ces $817 \mathrm{~km}$ exploitables, $707 \mathrm{~km}$ sont réellement exploités, soit $86,6 \%$. Les différences entre domaine exploitable et domaine exploité ne font pas ressortir de préférence particulière des pêcheurs pour certains milieux. 
Tableau II : Abondances relatives des différents milieux types dans les domaines exploitables et exploités en comparaison avec le domaine d'étude.

Table II : Relative abundance of the different environmental types in the potentially exploitable and exploited areas compared to the study area.

\begin{tabular}{crrr}
\hline MILIEU TYPE & $\begin{array}{c}\text { Domaine } \\
\text { d'étude }\end{array}$ & $\begin{array}{c}\text { Domaine } \\
\text { exploitable }\end{array}$ & $\begin{array}{c}\text { Domaine } \\
\text { exploité }\end{array}$ \\
\hline Bras principal & $38,2 \%$ & $41,1 \%$ & $41,8 \%$ \\
Retenue & $18,2 \%$ & $21,6 \%$ & $22,0 \%$ \\
Lit court-circuité & $11,3 \%$ & $9,8 \%$ & $11,3 \%$ \\
Canal & $10,2 \%$ & $8,9 \%$ & $8,5 \%$ \\
Bras secondaire & $3,7 \%$ & $2,4 \%$ & $1,8 \%$ \\
Affluent & $1,4 \%$ & $1,9 \%$ & $2,2 \%$ \\
Plan d'eau & $1,2 \%$ & $0,4 \%$ & $0,5 \%$ \\
Non décrit & $15,8 \%$ & $13,8 \%$ & $12,0 \%$ \\
\hline Total & $1105,5 \mathrm{~km}$ & $816,8 \mathrm{~km}$ & $707,1 \mathrm{~km}$ \\
\hline Fraction du domaine d'étude & $100 \%$ & $73,9 \%$ & $64,0 \%$ \\
\hline
\end{tabular}

Contrairement à ce que l'on observe dans les pêcheries du versant atlantique (ELIE et al., 1983 ; GABIGNON et al., 1984 ; ELIE et al., 1989), il n'existe pas d'autorisation administrative distinguant précisément les engins utilisés et les espèces recherchées. Seule la distinction entre pêcheurs amateurs et professionnels est généralisable à l'ensemble du bassin. Les différents métiers de pêche restent donc à définir pour ces deux catégories de pêcheurs. Le nombre maximum d'autorisations possibles semble déterminé de manière à maintenir un nombre constant de pêcheurs par kilomètre (corrélation positive significative avec la longueur du lot $r^{2}=0,67$ ) alors que le nombre d'admissions est indépendant de la taille du lot $\left(r^{2}=0,13\right)$. En réalité, le maximum possible d'autorisations n'est atteint que pour un lot sur trois et moins d'un lot sur deux dépasse la moitié de ce maximum possible. Cela est particulièrement sensible dans la Saône amont, le Rhône aval et le Rhône deltaïque où il n'y a manifestement pas d'adéquation entre les mesures administratives et la réalité de la pêche. Cela était déjà le cas sur la Loire au début de ce siècle (CARDAILLAC DE ST PAUL, 1902).

\section{MÉTHODES}

Les méthodes générales d'exploitation des statistiques de pêches proposent de ramener chaque quantité de poissons capturée à un effort de pêche élémentaire. Le rapport Capture par Unité d'Effort (CPUE) constitue la mesure la plus immédiate de l'abondance des captures (LAUREC et LE GUEN, 1981 ; LAUREC et al., 1983 ; WELCOMME, 1985). L'obtention de CPUE fiables demeure l'objectif essentiel de notre travail.

La collecte et le traitement des statistiques de pêches en milieu continental est confrontée à deux problèmes majeurs : d'une part, l'absence de site de débarquement précis où les productions sont centralisées pour d'éventuels prélèvements d'échantillons, d'autre part, une exploitation artisanale difficilement standardisable car elle fait intervenir plusieurs modes de pêche et porte sur plusieurs espèces simultanément.

Pour résoudre le problème d'échantillonnage, la déclaration écrite sur carnet de pêche est un procédé simple d'enquête qui a déjà été expérimenté avec succès en France sur les lacs alpins (GERDEAUX, 1988 ; GERDEAUX, 1990) et dans l'estuaire de la Gironde (ELIE et al., 1983 ; GABIGNON et al., 1984 ; ELIE et al., 1989). Notre document se présente sous la forme d'un calendrier sur lequel chaque pêcheur peut indiquer ses captures au jour le jour, généralement en poids par espèce. Les premières pages sont consacrées au dénombrement, à la description et à la précision des mois d'utilisation des engins. Les carnets sont remis et collectés chaque année lors du renouvellement des autorisations ou bien lors de la réunion annuelle des pêcheurs professionnels du bassin. Chaque carnet est ensuite intégralement saisi sur ordinateur. 
Les captures sont regroupées mensuellement pour limiter l'ampleur des données. L'ensemble de ces mesures n'a pas été rendu d'emblée obligatoire afin d'éviter au maximum les déclarations inexactes qu'on aurait pu attendre de l'application plus draconienne de la réglementation. Ces déclarations résultent d'une démarche volontaire qui dépasse les obligations administratives, ce qui limite la dissimulation des captures évoquée par ELIE et al. (1989). En revanche, elles restent incomplètes et les informations manquantes doivent faire l'objet d'une estimation au niveau des paramètres les plus simples de l'effort de pêche comme au niveau des captures.

Pour résoudre le problème posé par une pêcherie multi-spécifique et multi-engins, LAUREC et al. (1983) proposent de distinguer plusieurs métiers de pêche. Dans notre cas, la multiplicité des engins utilisés et des espèces recherchées pour un même engin nous empêchent de définir le métier simplement par l'association d'une technique de pêche et d'une espèce cible. Et cela d'autant plus que les espèces recherchées ne sont pas précisées dans les carnets. C'est pourquoi nous avons effectué une étude qualitative détaillée des caractéristiques des engins de pêche et de leur répartition spatio-temporelle pour orienter notre choix dans la distinction des métiers.

\subsection{Principes des estimations}

Les données manquantes se ramènent à deux cas : dans un premier cas, le carnet n'a pas été retourné ; dans un second cas, le carnet retourné n'est pas entièrement rempli.

\subsubsection{Estimations concernant les carnets non retournés}

Dans le premier cas, le nombre d'autorisations délivrées permet d'établir une estimation globale du nombre d'engins utilisés et des captures en utilisant la méthode de l'échantillonnage stratifié à chaque secteur d'exploitation (SCHERRER, 1983). Ceci dans l'hypothèse où les carnets retournés représentent un échantillon aléatoire simple de l'ensemble de pêcheurs d'une catégorie donnée dans l'un des cinq secteurs d'exploitation. Néanmoins, il est nécessaire de distinguer au préalable le nombre de réponses stricto sensu du nombre de carnets retournés dans le calcul du taux de réponses $f_{h}$. En effet, pour être comparés aux autorisations qui sont délivrées pour chaque pêcheur et chaque lot, certains carnets doivent représenter plusieurs réponses. Bien qu'étant théoriquement individuels, quelques carnets rendent compte de l'activité de plusieurs détenteurs d'autorisations qui pêchent généralement ensemble. II arrive également qu'un seul carnet rende compte de l'exploitation de plusieurs lots dans un même secteur d'exploitation. II faut donc retenir le nombre de lots et le nombre de pêcheurs concernés par un carnet pour connaître le nombre de réponses

$$
\begin{gathered}
n_{h}: \text { nombre de réponses annuelles pour le secteur d'exploitation } h \\
f_{h}=\frac{n_{h}}{N_{h}} \text { avec } \quad N_{h}: \text { nombre d'autorisations annuelles délivrées pour le secteur d'exploitation } h
\end{gathered}
$$

A ce taux de réponses, correspond un poids $W h$ du secteur d'exploitation retenu comme strate de l'échantillon:

$$
W_{h}=\frac{N}{N} \text { avec } \begin{gathered}
N_{h}: \text { nombre d'autorisations annuelles délivrées pour le secteur d'exploitation } h \\
N: \text { nombre total d'autorisations délivrées pour l'année }
\end{gathered}
$$

Les poids des secteurs permettent d'obtenir une estimation de la variable y (nombre de pêcheurs ou nombre d'engins) et de sa variance sur l'ensemble du domaine d'étude en appliquant les équations de l'échantillonnage stratifié à chaque secteur d'exploitation : 


$$
\begin{gathered}
\bar{y}=\sum_{h=1}^{5} W_{h} \bar{y}_{h} \text { et } v \overline{(y)}=\sum_{h=1}^{5} \frac{W_{h}^{2} s_{h}^{2}}{n_{h}}\left(1-f_{h}\right) \\
\overline{y_{h}} \text { : moyenne annuelle de la variable } y \text { dans la strate } h \\
s h \text { : écar type annuel de la variable } y \text { dans la strate } h \\
\hat{Y}=N \bar{y} \text { et } v(\hat{y})=N^{2} v(\overline{y)}
\end{gathered}
$$

L'intervalle de confiance de ces résultats se déduit de la variance de l'estimateur.

\subsubsection{Estimations concernant les carnets incomplets}

Ce second cas est pris en compte dans le calcul de la moyenne et de la variance de la variable $y$ à estimer dans chaque strate (secteur). Les carnets incomplets ont été considérés comme présentant une valeur de y moyenne pour la catégorie de pêcheurs et la strate considérée. Comme il n'y a pas de capture possible sans engins, les carnets déclarant des captures sans précision d'engin ont été considérés comme ayant un nombre d'engins moyen pour une strate et une catégorie de pêcheurs donnée. Les carnets sans pêche ont été ensuite inclus dans les calculs, ce qui conduit aux équations:

$$
\bar{y}_{h}=\frac{m}{\mathrm{M}} \bar{y}_{h}^{\prime} S_{h}^{2}=\frac{(m-1)}{\mathrm{M}} S_{h}^{\prime 2}+\frac{m(M-m)-\bar{y}^{\prime}}{\mathrm{M}^{2}}
$$

Avec pour chaque année et chaque strate $h$

$$
\begin{gathered}
\overline{y^{\prime}} \text { : nombre moyen d'engins déclarés } \\
S_{h}^{2}: \text { variance du nombre d'engins déclarés } \\
m: \text { nombre de carnets avec nombre d'engins ou captures déclarées } \\
M: \text { nombre de carnets total de la strate } h
\end{gathered}
$$

La stratification ultérieure sous-entend que la proportion de pêcheurs n'ayant pas pêché reste la même parmi ceux qui n'ont pas donné de réponse.

\subsection{Quantification de l'effort de pêche}

Le cahier des charges et les décrets préfectoraux fixent normalement le nombre de pêcheurs, les normes et le nombre des engins autorisés dans chaque lot pour chaque catégorie de pêcheurs, ainsi que les dates de fermeture de la pêche pour certaines espèces. D'une manière générale, les pêcheurs professionnels peuvent développer une plus grande puissance de pêche que les amateurs. Toutefois, les pratiques sont tellement diversifiées à l'échelle du bassin qu'il est difficile d'en avoir une vision globale. De plus, les pêcheurs utilisent rarement exactement tous les engins auxquels ils ont droit. Nous avons donc, dans un premier temps, effectué une quantification globale de l'intensité d'exploitation dans ses paramètres les plus simples (nombre d'autorisations, nombre de pêcheurs, nombre de jours de pêche). Puis, dans une seconde étape, nous avons étudié en détail les engins utilisés pour orienter notre définition des métiers. Ces études ont été effectuées suivant les méthodes statistiques exposées dans SAPORTA (1990) et CERESTA (1986) en utilisant ADE Software (CHESSEL et DOLEDEC, 1991). Tous les intervalles de confiance indiqués dans les résultats se rapportent au risque alpha de $5 \%$. 


\subsubsection{Mesure de l'intensité d'exploitation}

L'interprétation du nombre d'autorisations fluviales est délicate car il ne correspond pas exactement au nombre de pêcheurs (voir 3.1.1). Une estimation de ce nombre par catégorie de pêcheurs $i$ et par année $j$ est possible à partir du nombre moyen de pêcheurs par réponse $p_{i} / n_{i}$, calculé sur les deux années cumulées, ramené au nombre d'autorisations $N_{i j}$ :

$$
\widehat{P_{i j}}=\frac{P_{i}}{n_{i .}} N_{i j}
$$

L'effort de pêche peut être mesuré de différentes manières à partir de nos données suivant l'objectif recherché.

L'intensité de pêche annuelle, définie comme le nombre d'autorisations par kilomètre de linéaire délivrées annuellement dans chaque secteur d'exploitation donne un point de vue global de la répartition spatiale des pêcheurs, indépendant de la taille des secteurs.

L'intensité locale de pêche annuelle, définie comme le nombre d'autorisations délivrées annuellement par kilomètre de linéaire, calculée au niveau de chaque lot, situe les pêcheurs sur le linéaire indépendamment de la taille des lots. Son interprétation doit tenir compte du fait que l'action de pêche ne porte, en réalité, que sur certaines parties de ces lots.

L'intensité de pêche mensuelle, définie comme le nombre de pêcheurs déclarant au moins une capture pendant un mois donné, fait l'objet d'une estimation par échantillonnage stratifié par secteur d'exploitation exposée au 3.1.1. en prenant, pour chaque catégorie de pêcheurs et chaque mois, $y_{h}$ : le nombre de pêcheurs déclarant au moins une sortie dans la strate $\mathrm{h}$. Ce paramètre permet d'établir la répartition temporelle des pêcheurs.

\subsubsection{Typologie des engins déclarés}

En l'absence de renseignements concernant les espèces cibles, nous avons basé la définition des différents métiers de pêche sur l'étude qualitative des engins déclarés.

Pour décrire et rassembler les engins en types homogènes, nous avons retenu pour chaque engin déclaré :

- son appellation ;

- sa maille la plus petite qui conditionne généralement la taille des captures et rend compte de l'orientation de la pêche ;

- sa taille (longueur) ;

- son matériau de construction.

Dans un premier temps, l'appellation a permis d'identifier l'engin pour le ramener à l'une des grandes familles connues (DROIN DE BOUVILLE et DAUBREE, 1900 ; BRANDT, 1968 ; MONOD, 1973). Le nombre d'engins de chaque famille a fait l'objet d'une estimation annuelle par catégorie de pêcheurs suivant la méthode présentée au 3.1.1 et 3.1.2. Les équipements des deux catégories de pêcheurs ont été déterminés sur la base des différentes combinaisons de familles dans les réponses.

Dans un second temps, la maille et la taille des engins ont permis de distinguer des types au sein des familles les plus fréquentes. La réglementation est souvent intervenue dans le choix des classes de maille et de taille. Le matériau de construction a également été utilisé pour compléter la description. Le type et la famille se confondent pour les familles d'engins les moins fréquentes.

Une fois ces distinctions effectuées, les carnets déclarant l'usage d'un seul type d'engin ont été sélectionnés, puis les biomasses respectives des captures isolées. Pour un type d'engins donné, les captures placées en premier dans l'ordre d'abondance jusqu'à former une somme dépassant la moitié de la masse totale sélectionnée, ont été distinguées. L'établissement des rendements journaliers de chaque type d'engins a été effectué à partir d'une sous-sélection de carnets indiquant le nombre mensuel de jours avec captures et le nombre d'engins utilisés. 
Enfin, la somme de chaque type d'engins déclaré par mois, toutes catégories de pêcheurs confondues, a conduit au calendrier des pêches. De même, la somme de chaque type d'engins déclaré par secteur d'exploitation a permis d'établir leur répartition spatiale. Ces chiffres ont été ramenés à 100 carnets de manière à s'affranchir des variations spatiotemporelles de fréquentation relevant de l'intensité d'exploitation. Après élimination des types rares (moins de 10 engins déclarés pour cent carnets), les tableaux représentant les types d'engins en lignes et les mois en colonnes, ainsi que les types d'engins en lignes et les secteurs d'exploitation en colonnes ont été traités par Analyse Factorielle des Correspondances (AFC). Cette méthode élimine les différences d'effectifs entre les lignes (types d'engins) qu'il est inutile de conserver car elle sont déjà connues au travers des estimations effectuées précédemment pour chaque famille. Les types d'engins ayant une contribution absolue supérieure à $10 \%$ de l'inertie du plan ont été retenus comme lignes structurantes dans chaque analyse.

\section{RÉSULTATS}

Pendant ces deux années d'exploitation, il a été délivré en moyenne 712,5 autorisations amateurs par an et 91,5 autorisations professionnelles par an, soit un total de 1608 autorisations sur deux ans.
Tableau III : Caractérisation des sources d'information en termes de précision des données. Répartition des réponses suivant l'année (1988 ou 1989), la catégorie de pêcheurs (Amateurs ou Professionnels) et les indications qu'elles contiennent concernant les captures, les engins et le nombre de jours de pêche mensuel.

Table III : Characterization of data sources in terms of the accuracy of reporting for 1988 and 1989 . Information was collected from commercial and recreational fishermen on species captured, gear used and number of days fished per month.

\begin{tabular}{cccccccc}
\hline & INDICATIONS & \multicolumn{3}{c}{1988} & \multicolumn{3}{c}{1989} \\
\hline Captures & Engins & Nb de jours & Amateurs & Professionnels & Amateurs & Professionnels & TOTAL \\
\hline précisées & précisés & précisés & 199 & 33 & 210 & 21 & 463 \\
précisées & précisés & non précisés & 33 & 17 & 29 & 15 & 94 \\
précisées & non précisés & précisés & 21 & & 29 & 6 & 56 \\
précisées & non précisés non précisés & 12 & & 6 & 3 & 21 \\
non précisées & précisés & non précisés & 5 & & 8 & & 13 \\
non précisées & non précisés non précisés & 44 & 5 & 49 & 7 & 105 \\
\hline \multicolumn{2}{r}{ NB TOTAL } & & 314 & 55 & 331 & 52 & 752 \\
\hline NB AUTORISATIONS & 703 & 97 & 722 & 86 & 1608 \\
\hline
\end{tabular}

Sur les 711 carnets reçus, seulement 6 carnets amateurs rendent compte de l'activité de plusieurs pêcheurs. La distinction réponse-carnet est importante surtout pour les professionnels qui remettent un seul carnet pour plusieurs lots (en moyenne 2,5 $\pm 0,2$

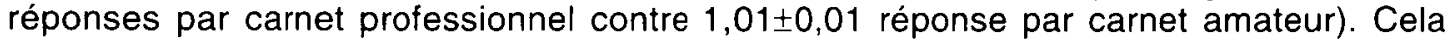
porte à $46,8 \%$ le taux de réponses, catégories de pêcheurs, années et secteurs confondus (Tableau III). Ce taux peut localement s'annuler, ce qui empêche les calculs d'estimation à l'échelle du lot. Néanmoins, au niveau du secteur, ce taux peut dépasser $60 \%$. Chez les amateurs (Fig. 3a), il est stable d'une année sur l'autre et augmente clairement avec le nombre d'autorisations délivrées pour suivre une allocation proportionnelle. En revanche, cet effet de masse ne se retrouve pas chez les professionnels en raison d'un taux de réponses particulièrement élevé dans le Rhône deltaïque (Fig. 3b). 
Une proportion non négligeable de réponses $(15,7 \%)$ ne signale aucune capture. Ces carnets précisent généralement qu'aucune pêche n'a été effectuée. Ce type de réponses progresse légèrement entre 1988 et 1989 (Tableau III).

\subsection{Intensités de pêche}

Entre 1988 et 1989 le nombre estimé de pêcheurs professionnels passe de 39 à 35 et celui des amateurs de 698 à 717 . Il y a donc en moyenne 20 amateurs pour 1 professionnel.

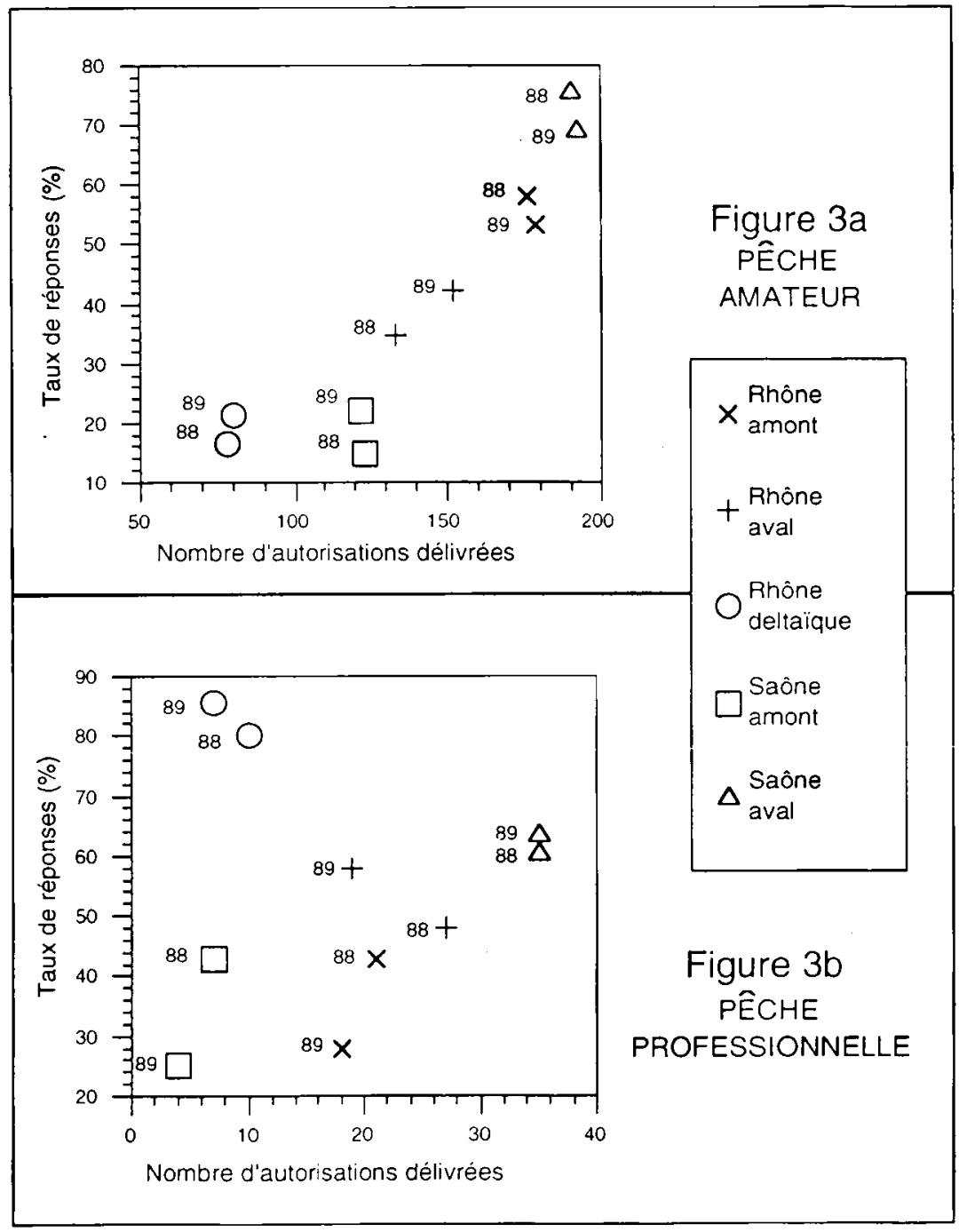

Figures 3a et 3b : Taux de réponses en fonction du nombre d'admissions par secteur d'exploitation. Les chiffres indiquent les années. Contrairement aux professionnels (3b), pour un secteur donné, les réponses des amateurs (3a) augmentent avec le nombre de pêcheurs admis.

Figures $3 a$ and $3 b$ : Response rates ploted against the number of fishermen admitted in each exploitation sector. The numbers indicate the years. In contrast to commercial fishermen (3b), the response rate of the recreational fishermen in a given exploitation sector (3a) increase with the number of fishermen admitted. 


\subsubsection{Intensité de pêche annuelle}

L'analyse de variance de l'intensité de pêche annuelle (en nombre d'autorisations $/ \mathrm{km} / \mathrm{secteur} / \mathrm{an}$ ) montre que :

- le secteur d'exploitation intervient de manière très significative (test-F où $p<0,005)$;

- les deux années d'exploitation sont semblables $(F<1)$, ce qui justifie le calcul de moyennes dans la représentation graphique (Fig. 4).

Chez les amateurs, l'intensité de pêche annuelle permet de classer les secteurs dans l'ordre décroissant : Saône aval, Rhône amont, Rhône deltaïque, Rhône aval et Saône amont. Elle est particulièrement basse pour ces deux derniers secteurs d'exploitation qui ne sont pas significativement différents entre eux (test de Scheffe à $95 \%$ ).

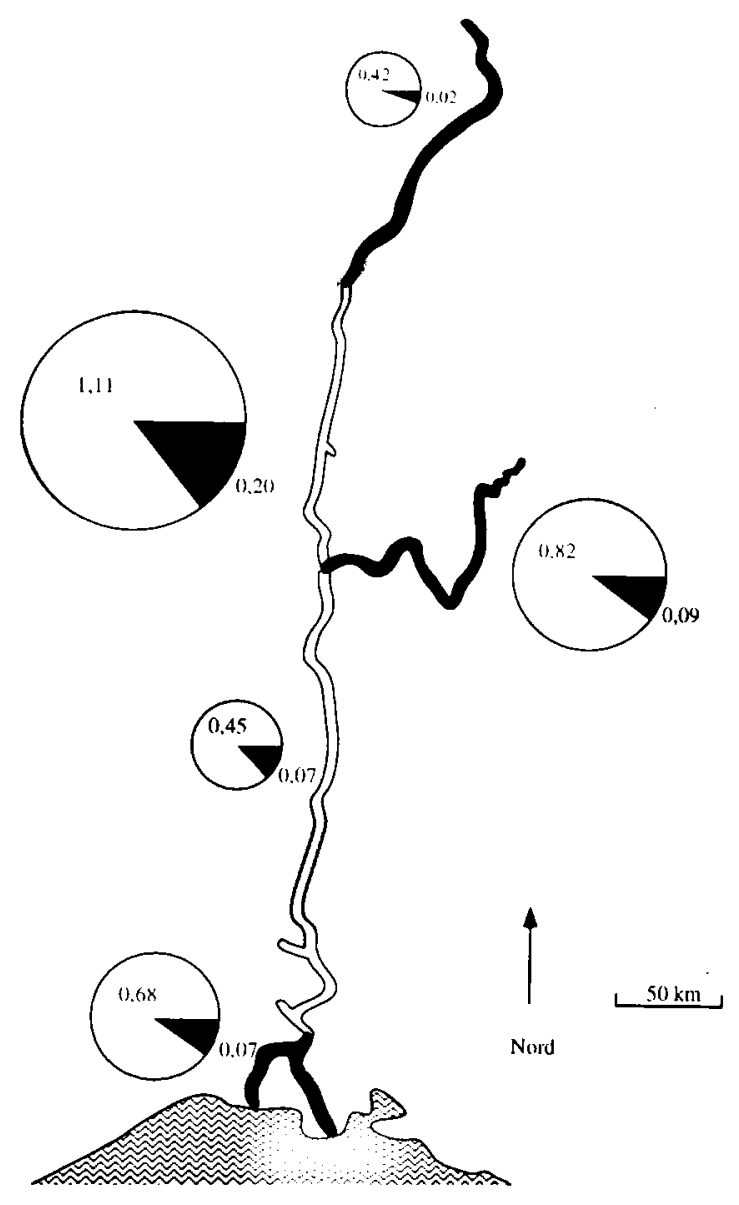

Figure 4 : Moyennes sur deux ans (1988 et 1989) de l'intensité de pêche annuelle (pêcheur $/ \mathrm{km}$ ) pour chaque secteur d'exploitation. Les diamètres des cercles sont proportionnels à la somme des densités, les amateurs sont figurés en blanc, les professionnels en noir.

Figure 4 : Fishing intensity averaged over the years 1988 and 1989 for each exploitation sector (units : fishermen $/ \mathrm{km}$ ). The diameters of the circles are proportional to the sum of the densities, the non-commercial licenses in white, the commercial licenses in black. 
Chez les professionnels, la Saône aval se distingue de tous les autres secteurs d'exploitation avec une valeur plus élevée. Puis le Rhône amont, le Rhône deltaïque, le Rhône aval et la Saône amont se succèdent suivant un ordre décroissant avec un continuum de différences non significatives deux à deux (test de Scheffe à $95 \%$ ).

\subsubsection{Intensité locale de pêche annuelle}

L'intensité locale de pêche annuelle peut atteindre 6,8 et 1,1 autorisation $/ \mathrm{km} / \mathrm{lot} / \mathrm{an}$ respectivement pour les amateurs et les professionnels. Elle ne suit pas une loi normale ( $p \leq 0,01$ bilatéral avec le test de Lilliefors in CHESSEL et DOLEDEC, 1991). Les ajustements à la loi de Poisson sont mauvais (Chi-deux $p<0,001$ ). La dispersion des autorisations n'est donc pas aléatoire, mais agrégative. Globalement, elle reste stable d'une année sur l'autre (test de corrélation des rangs de Kendall hautement significatif ( $p \leq 0,0001$ bilatéral)). Toutefois, prise séparément, l'intensité locale de pêche annuelle des professionnels diminue entre 1988 et $1989(p \leq 0,004$ unilatéral au test des séries appariées de Wilcoxon). Par contre, elle présente des distributions différentes suivant les secteurs ( $p \leq 0,0009$ au test de Kruskal et Wallis) :

Chez les amateurs, l'intensité locale de pêche annuelle permet de classer les secteurs dans l'ordre décroissant : Saône aval, Rhône amont, Rhône deltaïque, Rhône aval et Saône amont. Elle est particulièrement basse pour ces deux derniers secteurs bien qu'il existe un continuum de différences non significatives deux à deux ( $p>0,05$ au test $U$ de Mann et Witney).

Chez les professionnels, elle permet de scinder les secteurs en trois groupes ( $p \leq 0,05$ au test $U$ de Mann et Witney deux à deux) avec dans l'ordre décroissant : le Rhône deltaïque, la Saône aval, le Rhône amont, puis le Rhône aval, puis la Saône amont.

\subsubsection{Intensité de pêche mensuelle}

L'intensité de pêche mensuelle estimée (en nombre de pêcheurs/mois) est obtenue avec une erreur relative variant de 9 à $14 \%$ au risque alpha de $5 \%$ suivant l'année et la catégorie de pêcheurs. Elle suit un rythme saisonnier chez les deux catégories de pêcheurs avec un minimum en mai durant la fermeture partielle de la pêche (Fig. 5).

La fréquentation maximum des sites de pêche se situe en saison "été-automne" entre juin et novembre. A partir de décembre et jusqu'en avril, un niveau de fréquentation plus bas s'établit suivant une saison "hiver-printemps". Le contraste hydraulique entre 1988 et 1989 permet de montrer l'influence du débit sur ce rythme saisonnier. En 1988, durant la saison hiver-printemps, le débit mensuel particulièrement important mesuré à proximité de l'émissaire du bassin est associé à une fréquentation plus faible qu'en 1989 par les pêcheurs amateurs (Fig.2 et Fig. 5 ). L'augmentation de ce débit fait généralement baisser le nombre de pêcheurs (pente significativement négative $p \leq 0,0015$, mois de fermeture exclu).

\subsection{Familles et types d'engins}

570 réponses déclarent les engins utilisés, soit $89,9 \%$ des réponses reçues si on exclut les réponses sans pêche (Tableau III). Un pêcheur sur dix ne précise pas les engins qu'il utilise tout en déclarant des captures. Dans l'estimation du nombre total d'engins sur le bassin, ces pêcheurs sont considérés comme étant équipés d'un nombre moyen d'engins pour leur catégorie dans le secteur (strate) considéré.

A partir des quarante appellations différentes observées, nous avons pu établir sept familles différentes (en colonnes dans le Tableau IV). La représentation de chaque famille dans les réponses reste stable d'une année sur l'autre (corrélations inter-annuelles $\left.r^{2}=0,98\right)$. Les professionnels se caractérisent par un nombre d'engins beaucoup plus élevé que celui des amateurs. Ce nombre est connu avec une faible précision. Néanmoins, il décroît significativement dans presque toutes les familles entre 1988 et 1989 . 


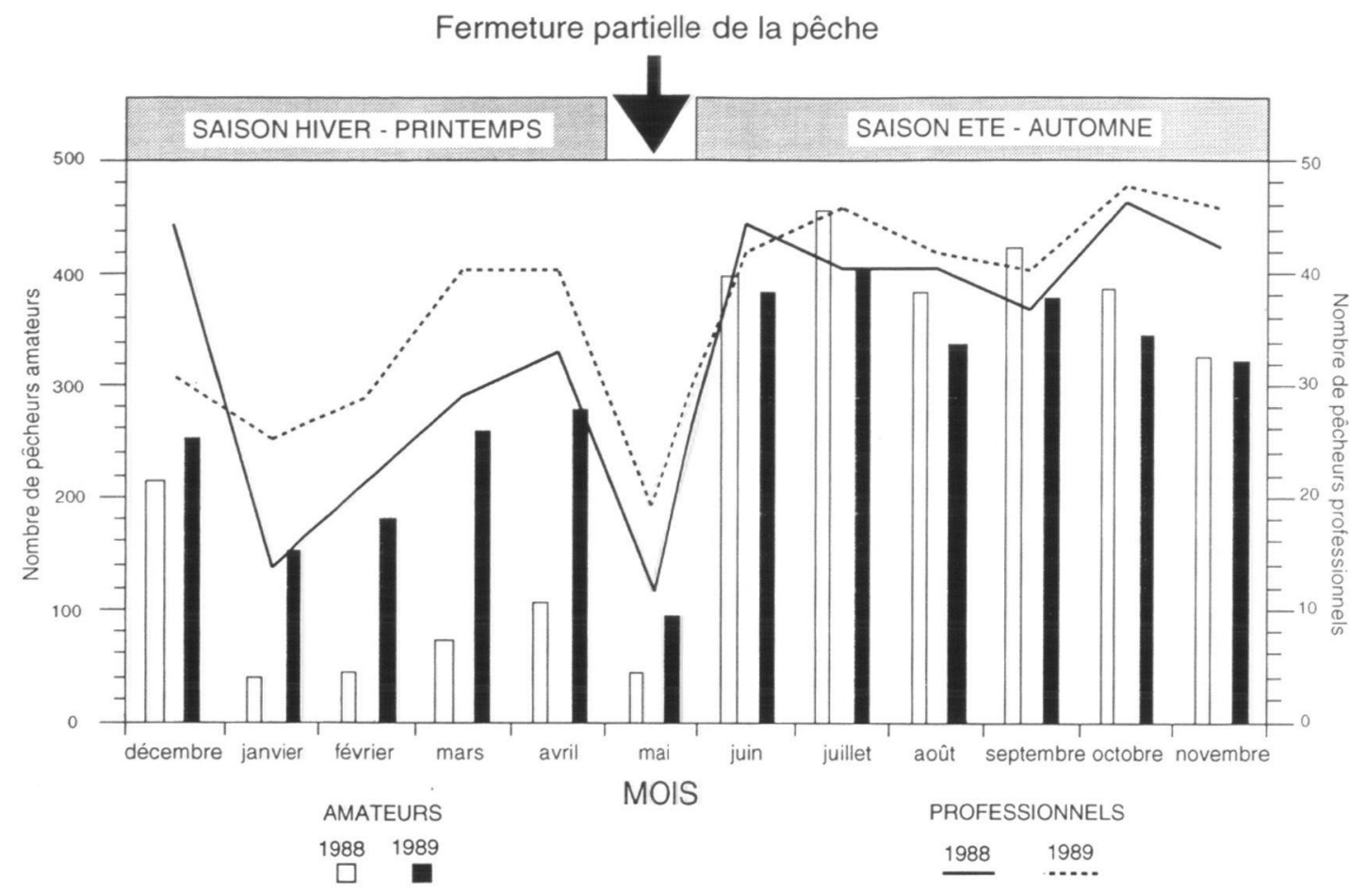

Figure 5 : Intensité de pêche mensuelle estimée (nombre total de pêcheurs sur le domaine d'étude) pour la pêche professionnelle (lignes) et amateur (histogrammes) durant les années 1988 et 1989. On distingue deux saisons séparées par une fermeture partielle de la pêche.

Figure 5 : Estimates of monthly fishing intensity (total number of fishermen on the studied area) for commercial (lines) and recreational (bars) fishing during 1988 and 1989. Two seasons are apparent, separated by a partial closure of the fishery.

Les combinaisons de familles les plus fréquentes (Tableau IV) font ressortir chez les deux catégories de pêcheurs l'usage d'une famille unique d'engins : les carrelets chez les amateurs, les pièges fixes chez les professionnels. L'utilisation exclusive de filets arrive en deuxième position chez les deux catégories de pêcheurs. Puis, suivent les équipements à deux, trois, voire quatre familles d'engins différentes. Les amateurs présentent, à ce titre, une diversité plus grande. Leur équipement peut se composer de 29 combinaisons de familles d'engins différentes alors qu'ils n'utilisent pas de senne, ce qui limite à 63 le nombre de combinaisons théoriquement possibles (somme des combinaisons de 1 à 6 familles parmi 6). De leur côté, les professionnels ne déclarent que 14 combinaisons différentes alors qu'ils utilisent les sept familles d'engins (soit 106 possibilités en théorie).

Le rendement moyen journalier permet de classer les familles dans l'ordre croissant : pièges fixes, carrelets, tramails ou filets (Tableau $V$ ).

Sur les sept familles d'engins, les sennes, les éperviers et les lignes étaient trop peu nombreux pour faire l'objet d'une distinction plus précise. La distribution des types de pièges, de filets et de carrelets en fonction de la maille et de la taille montre de nombreux engins dans la diagonale (Fig. 6), ce qui est le signe d'une corrélation entre ces deux paramètres. Les grandes longueurs sont généralement réservées aux professionnels (toutes les trames de la Fig. 6). La valeur maximum de la maille progresse régulièrement depuis les pièges jusqu'aux filets (histogrammes noirs de la Fig. 8) suivant le mème ordre que celui établi par les rendements (Tableau V). Au sein de chaque type, les rendements augmentent avec la maille. 
Tableau IV : Nombre total d'engins estimé sur le domaine étudié pour les amateurs, au dessus, et pour les professionnels, en dessous. Chaque ligne représente une combinaison d'engins rentrant dans l'équipement avec, à gauche, le nombre de réponses correspondantes. Le nombre moyen de chaque famille d'engins est précisé dans la partie droite. Tous les intervalles de confiance sont indiqués au risque alpha de $5 \%$.

Table IV : Total number of fishing gear estimated in the study area for commercial fishermen (upper table) and for recreational fishermen (below). Each line corresponds to the combination of fishing gear with, on the left, the associated number of responses. The corresponding average number of fishing gear is given on the right. All confidence intervals are given at an alpha value of $5 \%$.

\begin{tabular}{|c|c|c|c|c|c|c|c|}
\hline \multirow{2}{*}{$\begin{array}{l}\text { Sumbere de réponses } \\
\text { sur deux anneses dexploitation }\end{array}$} & \multicolumn{7}{|c|}{ FQLUPFLAFNT ANATELK } \\
\hline & Carrelets & Preasiers & Filsks & Lignes & Pregesda & $\therefore \cdot m i n$ & I:_:mal:- \\
\hline 129 & $1.36 \pm 0.07$ & & & & & & \\
\hline 83 & & & $1.4 \pm 0.2$ & & & & \\
\hline 80 & $1.7 \pm 0.3$ & & & & $7 \pm 1$ & & \\
\hline 70 & & & & & $5.7 \pm 0 . ?$ & & \\
\hline 29 & & & $1,4 \div 0,3$ & & $2.5 \pm 0.2$ & & \\
\hline 43 & & & pour 24 & ulles com & naisone & & \\
\hline 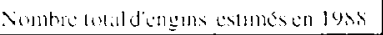 & 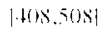 & $15.5(1)$ & $I=96,3964$ & $1+1:(9) !$ & $1112: 14 \div 41$ & & $\cdots, \bar{\vdots}$ \\
\hline 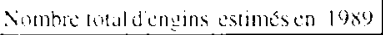 & $14(1-2 \cdot+9 ! 1$ & $|1+4 ; 4|$ & $1246, .39+1$ & $1+1,1060 \mid$ & $11237.1,3.451$ & & $1: 2-4$ \\
\hline
\end{tabular}

\begin{tabular}{|c|c|c|c|c|c|c|c|}
\hline \multirow{2}{*}{ 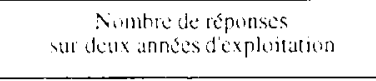 } & \multicolumn{7}{|c|}{ HQLIPHAINTT PROHESSIONVLL } \\
\hline & Carreless & Pporvies & rikns & Lignas & PligL lixs & sines & 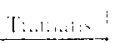 \\
\hline 23 & & & & & $57 \pm 73$ & & \\
\hline 18 & & & $7 \pm 6$ & & & & \\
\hline 15 & & & $12 \div 4$ & & $152+110$ & & ! $\cdots, 1$, \\
\hline 8 & & & $9 \pm 4$ & & $60 \pm 50$ & & \\
\hline 32 & \multicolumn{7}{|c|}{ pont lo antios combinatsons } \\
\hline Nombre cotaldengins estimes en 1988 & $133 ; 791$ & $111: 211$ & $1552 ; 7181$ & $17: 71$ & $1.32615: 60000$ & $|1: 5|$ & $1 \cdot 4+:-7 \mid$ \\
\hline Nombre cotaldengins estimés an 1989 & 125,511 & $15: 111$ & $1414: 5241$ & 12.51 & $120,54,460.41$ & & $1: 2: 321$ \\
\hline
\end{tabular}

\subsubsection{Les pièges fixes}

La famille des pièges fixes est numériquement la plus importante (Tableau IV), mais globalement sa représentation dans les réponses est plus faible que celle des filets (287 réponses contre 360 ). Cela tient au fait qu'ils sont toujours utilisés en grand nombre. Dix-neuf appellations différentes se distinguent dans les réponses. Nous avons retenu trois types:

Le type 1 rassemble les pièges de grandes tailles et petites mailles (en tramé noir sur la Fig. 6 et les Tableaux $V$ et $V I$ ), construits en filets dits nasses anguillères, cerfsvolants ou ganguis ; ils sont utilisés uniquement par les professionnels pour la pêche de l'anguille (Anguilla anguilla) avec l'anguille argentée de fin septembre à fin décembre et l'anguille sédentaire d'avril à juillet.

Le type 2 rassemble les pièges de tailles variables et de mailles moyennes à grandes (en noir sur la Fig. 6 et les Tableaux V et VI). On distingue les nasses à poissons, grandes nasses, nasses hexagonales, nasses plates en filet rigide des varveux, ou verveux construits en filet souple ; ils capturent surtout du poisson-chat (Ictalurus melas), de la perche (Perca fluviatilis) et du barbeau fluviatile(Barbus barbus).

Le type 3 rassemble les pièges de petites tailles et de petites mailles (en gris sur la Fig. 6 et les Tableaux $V$ et VI) généralement faits de grillage rigide, dits nasses anguillères, bosselles, casiers, nasses carrées, petites nasses, nasses à barrettes ou nasses à écrevisses ; ils capturent surtout du poisson-chat et de l'écrevisse (Orconectes limosus). 
Tableau V : Partie gauche : captures obtenues sur des extraits se rapportant à l'emploi d'un seul type d'engin. Les captures principales représentent $50 \%$ du total extrait.

Partie droite : rendements journaliers moyens de chaque type d'engin ("ND" pour Non Déterminé).

Table V : On the left : catches obtained from a sampling of a single fishing gear type. Principal catches are those which comprise the first $50 \%$ of the selection (ordered by decreasing weight of total catch).

On the right : average daily catch rate for each type of fishing gear ("ND" for Not Determined).

\begin{tabular}{|c|c|c|c|c|c|c|}
\hline FAMILLE & TYPE & $\begin{array}{l}\text { LEGENDE } \\
\text { Fig. } 8\end{array}$ & $\begin{array}{l}\text { Tonnage } \\
\text { extrait }\end{array}$ & Captures principales & $\begin{array}{l}\text { Kilos } \\
\text { extraits }\end{array}$ & $\begin{array}{c}\text { Rendement moyen } \\
(\mathrm{kg} /(\text { engin } \mathrm{x} \text { journee })\end{array}$ \\
\hline Pièges fixes & 1 & & 8.7 & anguille & 0 & \\
\hline Picges fixes & 2 & & 11.9 & poisson-chat, perche, bartea lluviatile & 837 & $0.8 \pm 0.3$ \\
\hline Piiges tixes & 3 & 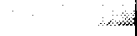 & 0,9 & poisson-chall, Ecrevisse ank ricaine & 720 & $0.6 \pm 0.1$ \\
\hline Pieges fixes & aulres & & $<0.1$ & anguillc & 8 & $0.9 \pm \mathrm{N}()$ \\
\hline Piiges fixes & non décrits & & 0.7 & poisson-chal, serevisse américaine, perche & 487 & $0.7 \pm 0.3$ \\
\hline Filets & 1 & & 0.1 & barterau fluvialile. holu & 23 & $10: 161$ \\
\hline rilets & 2 & & 0 & & 0 & \\
\hline filcts & 3 & & 5.7 & hott. gardon. harbeau flusiatile & 3267 & $10 \pm 2$ \\
\hline Filets & 4 & & 11,6 & muge, holu, brème sp. & 7370 & $2.3 \pm x$ \\
\hline Filets & 5 & & 0.5 & horu, sandre & 2.54 & $10: 351$ \\
\hline Filees & 6 & & 2.6 & alose feinte. brème sp. & 352 & $29 \pm 14$ \\
\hline Files & autres & & 0 & & () & \\
\hline filcess & non décrits & & 0.4 & holu & 0 & \\
\hline Canclets & 1 & & 0.2 & ablelle & 92 & $2.7 \pm 0.7$ \\
\hline Curreless & 2 & 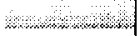 & 9.2 & silure, hremc sp.. sandre, carpe & 4942 & $5.5 \pm 0.5$ \\
\hline Carreless & autres & & 2.7 & alose feinte. hreme sp.. muge & 1797 & $11 \pm 4$ \\
\hline Carrelets & non dscrits & & 1.4 & grands cyprinides. sandre, giardon & 372 & $6 \pm 2$ \\
\hline Tramails & 1 & & 0.1 & poisson-chat, brèmc sp. & 89 & $15 \pm 12$ \\
\hline Tramails & autres & & 0 & & 0 & \\
\hline Tramails & non decrits & & 0 & & 0 & \\
\hline Lignes & & & 0,1 & bartheau fluviatile, truile & 0 & \\
\hline Fperviers & & & $<0,1$ & friture & 5 & $|0: 2|$ \\
\hline Sennes & & & 0 & & 0 & \\
\hline
\end{tabular}

\subsubsection{Les filets maillants}

Les filets maillants représentent la famille la plus répandue dans les réponses. Elle rassemble six appellations différentes dérivées du synonyme araignée. Distinguer des types précis selon nos critères est ici plus difficile car il s'agit aussi de la famille la plus diversifiée, en maillages comme en tailles. Nous avons retenu comme limite de taille les $30 \mathrm{~m}$ de longueur maximum autorisés pour les amateurs sur la majorité du bassin. Les classes extrêmes de maillages ont été ensuite séparées. II en résulte six types:

Les types 1 et 2 (en noir et tramé blanc sur fond noir sur la Fig. 6 et les Tableaux $V$ et $\mathrm{VI}$ ) correspondent aux filets à petites mailles ; la biomasse des captures contenue dans les carnets utilisant uniquement ce type de filets $(23 \mathrm{~kg})$ n'est pas suffisante pour être retenue comme échantillon représentatif.

Les types 3 et 4 (en gris et tramé normal sur la Fig. 6 et les Tableaux $V$ et VI), correspondent aux filets de mailles moyennes; ils capturent surtout du muge (Liza ramada en majorité) et des cyprinidés (hotu, Chondrostoma nasus), brèmes sp. (Abramis brama et Blicca bjoerkna), barbeau fluviatile, gardon (Rutilus rutilus).

Les types 5 et 6 (en gris clair et tramé espacés sur la Fig. 6 et les Tableaux $V$ et VI) correspondent aux filets de grands maillages pour la pêche des mêmes espèces que précédemment, auxquelles s'ajoutent l'alose feinte (Alosa fallax) et le sandre (Stizostedion lucioperca). 


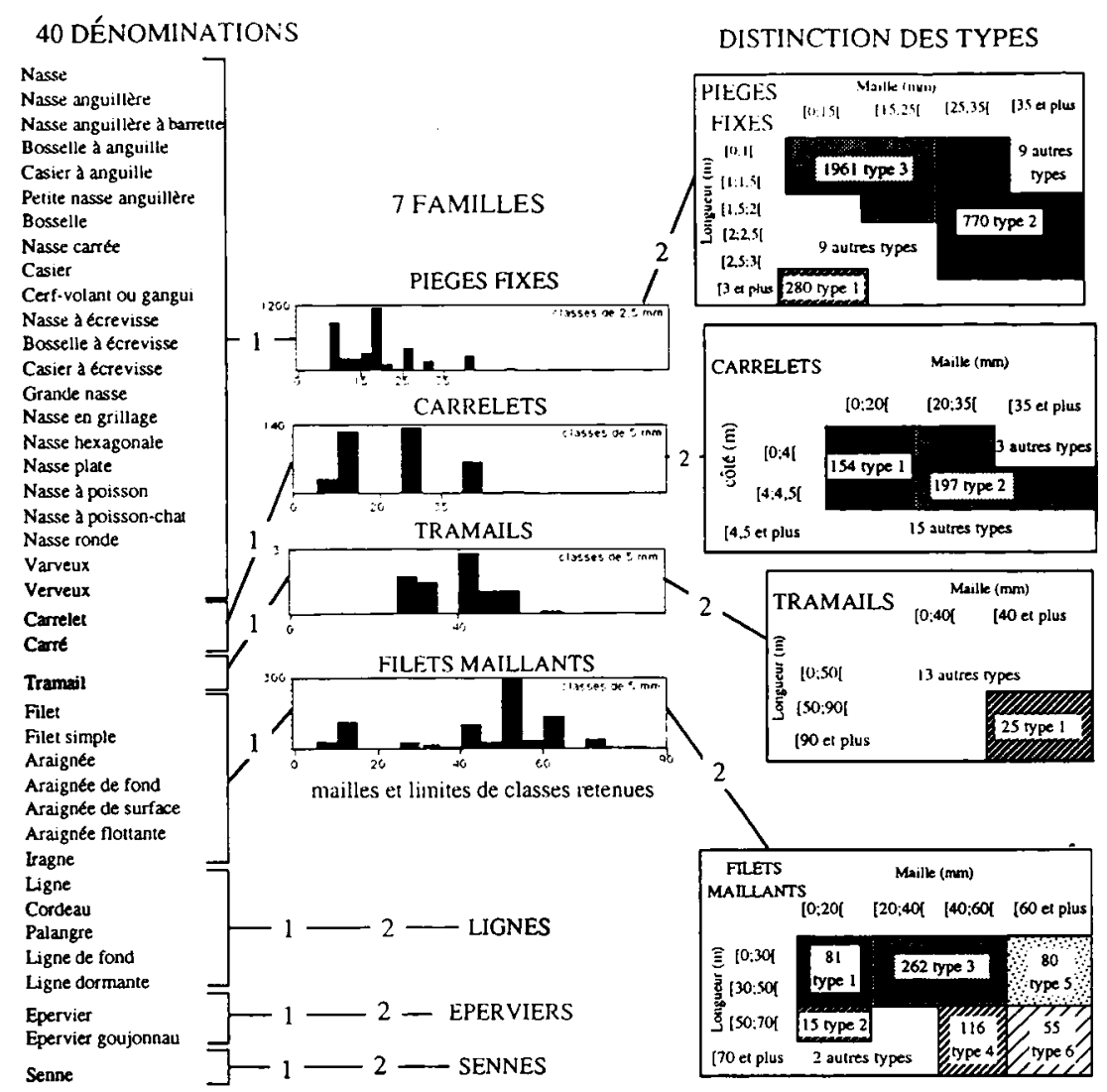

Figure 6 : Classification des engins déclarés :

1 - détermination des familles à partir des dénominations ;

2 - distinction des types à partir du maillage et de la taille des familles d'engins les plus représentés (les plans F1 X F2 des lignes de l'ACPn sont représentés le cas échéant, chaque type est figuré par une trame différente, le nombre d'engins de chaque type est précisé dans le tableau final à double entrée).

Figure 6 : Classification of the declared fishing gear :

1 - determination of families according to denominations ;

2 - the characterization of types distinguished by mesh size and length (factorial map F1 X F2 of Principal Component Analysis on correlation matrices are represented when necessary, each type having a different frame and each being identified on the final table).

\subsubsection{Les carrelets}

La famille des carrelets est essentiellement d'un usage amateur (Tableau V). Le côté maximum admis par la réglementation est de $4 \mathrm{~m}$ sur la majorité des lots considérés. On peut distinguer deux types de carrelets :

Le type 1 rassemble les carrelets à petites mailles (en noir sur la Fig. 6 et les Tableaux V et VI), destinés à capturer "la friture" d'ablette (Alburnus alburnus);

Le type 2 rassemble les carrelets à plus grandes mailles (en gris sur la Fig. 6 et les Tableaux $V$ et $\mathrm{VI}$ ), utilisés pour la pêche du silure (Silurus glanis), des brèmes sp. et du sandre ;

Un petit nombre de carrelets hors gabarit (en blanc sur la Fig. 6 et les Tableaux $V$ et $\mathrm{VI}$ ) comprennent ceux qui servent à capturer des aloses feintes. 


\subsubsection{Les tramails}

La familie des tramails arrive en troisième position, devant les carrelets chez les professionnels alors qu'elle est rarement utilisée par les amateurs (Tableau IV). Les grandes tailles et mailles constituent le type 1 (en tramé noir sur la Fig. 6 et les Tableaux $V$ et $V I$ ). Les quantités de captures disponibles ne permettent pas de connaître avec précision leur composition.

\subsubsection{Les lignes, les éperviers et les sennes}

Les lignes dormantes, cordeaux, lignes de fond ne sont pratiquement pas utilisées par les professionnels. Elles apparaissent en petit nombre chez des amateurs. Elles sont plus sélectives que les filets pour la pêche des poissons de fond ou à régime carnivore (barbeau fluviatile, truite (Salmo trutta)). friture".

Peu nombreux, les éperviers de petites mailles (9 à $10 \mathrm{~mm}$ ) sont utilisés pour "la

De même, une seule senne a été déclarée pendant ces deux années. Cet engin à pratiquement disparu à moins que certains filets ou tramails, dont le mode d'utilisation est rarement précisé, soient maniés en sennes.

Tableau VI : A gauche : distribution temporelle des engins déclarés par types et familles cumulés sur l'ensemble du domaine d'étude durant les années 1988 et 1990.

A droite : distribution spatiale suivant les différents secteurs d'exploitation. Le nombre d'engins est ramené à 100 carnets pour un mois ou un secteur d'exploitation donné. Le nombre de carnets considérés et la proportion de pêcheurs professionnels sont précisés en dessous.

Table VI : On the left : temporal distribution of the declared fishing gear listed by type and family over the whole study area for the years 1988 and 1990.

On the right : spatial distribution of the declared fishing gear in the different exploitation sectors. The number of fishing gear is taken from the contribution of $100 \mathrm{log}$-books for a given month or exploitation region. The actual number of log-books and the proportion of commercial fishermen are listed at the bottom.

\begin{tabular}{|c|c|c|c|c|c|c|c|c|c|c|c|c|c|c|c|c|c|c|c|}
\hline FAMILLE & TYPE & $\begin{array}{c}\text { I.FGENDF } \\
\text { Fig. }\end{array}$ & an & fer & mar & avr & $\mathrm{maj}$ & MOIS & jul & Dol & sep & nct & nov & dec & $\begin{array}{l}\text { Rhone } \\
\text { amon: }\end{array}$ & $\begin{array}{c}\text { Rhone } \\
\text { aval }\end{array}$ & $\begin{array}{c}\text { Rhone } \\
\text { deltä̈que }\end{array}$ & $\begin{array}{l}\text { Sabne } \\
\text { amont }\end{array}$ & $\begin{array}{l}\text { Sabne } \\
\text { avid }\end{array}$ \\
\hline Pièges fixes & 1 & & & & & 109 & 126 & 51 & 45 & 12 & 71 & 81 & 87 & 89 & & 201 & 343 & & \\
\hline Pieges fixes & 2 & & 495 & 476 & 365 & 297 & 277 & 228 & 213 & $2(x)$ & 219 & 256 & 258 & 299 & (11) & 22 & 18 & 76 & 244 \\
\hline Pieges fixes & 3 & & 2622 & 2565 & 1968 & 1584 & 16,36 & 988 & 872 & 885 & 887 & 1008 & 1082 & 1,301 & $s$ & 11 & 155 & 16 & 79)7 \\
\hline Pieges fixes & Jutres & & & & & 3 & & 4 & 4 & 4 & $s$ & $s$ & 5 & 6 & & 12 & & & 4 \\
\hline Piters fixes & nun dicilis & & 979 & 961 & 751 & 570 & (64) & 433 & 305 & $f(x)$ & 414 & 3ys & H: & 529 & (i) & 17 & 132 & 85 & bos \\
\hline Filets & I & & & & & & 37 & 39 & 36 & $w_{1}$ & 10 & $s$ & 3 & 3 & 3 & & & 16 & 11 \\
\hline Fikers & $=$ & & & & 5 & 9 & & 9 & ) & 1) & 6 & 7 & 4 & & & & & & 7 \\
\hline Filets & $x$ & & 5i) & 42 & 57 & \$4 & $\$$ & $\$ 1$ & +1 & +1 & 41 & 45 & 48 & 4.3 & $1 \times 1$ & 43 & & 47 & w1 \\
\hline |ijels & + & & 97 & 101 & $n$ & 37 & 41 & 28 & 27 & 28 & 43 & 48 & Sit & (x) & 23 & 21 & 12 & 16 & 25 \\
\hline Fibless & 5 & & 35 & 4 & 27 & 22 & 6 & 13 & 12 & I: & 17 & 19 & 18 & 21 & 9 & 39 & & 16 & 14 \\
\hline Filets & a & & 8 & 13 & 10 & 11 & $1:$ & 6 & 3 & 3 & 8 & 9) & 10 & 12 & 1 & 4 & 18 & & is \\
\hline Filiets & sutis: & & & & & & & & & : & 2 & & & & & 3 & & & \\
\hline linhers & non dicialk & & 2 & & & & & & 1 & & 1 & 1 & & & 2 & & & + & 4) \\
\hline Cuntelets & 1 & & $\therefore 1$ & 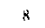 & $y$ & 9 & 11 & 24 & 24 & $\approx x$ & 25 & 22 & 13 & 11 & 16 & 4.3 & & 4 & +1 \\
\hline Cumelets & 2 & & & 27 & 28 & 30 & 24 & 28 & 22 & 24 & 22 & 22 & 26 & 28 & 6 & 9 & 49 & $\therefore$ & $x_{1}$ \\
\hline Cantikets & sutris: & & $=$ & 4 & 6 & a & 6 & ? & $z$ & 2 & 2 & 1 & 1 & 1 & 1 & 6 & 23 & & $:$ \\
\hline Carrikets & nun dients & & & & & & & ? & 4 & 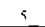 & 6 & 5 & 5 & 6 & 4 & 8 & 4) & 1,4 & 1.5 \\
\hline Framuils & 1 & & 11 & 11 & 1!1 & 9 & 12 & 11 & 9 & 9 & 8 & 9 & 10 & 10 & & 2 & 6 & 29 & a \\
\hline Tramiults & Jutres & & 4 & 4 & 5 & 4 & 3 & 3 & 3 & 3 & 3 & 4 & 4 & 4 & 3 & & 3 & 11 & 3 \\
\hline Tramialis & nun dicnts & & & & & & & 1 & 1 & 1 & 1 & 1 & 1 & & & & & $?$ & 2 \\
\hline Lignes & & & 8 & & $=$ & $\approx$ & 8 & $y$ & 12 & 11 & 5 & 7 & a & 1 & 1.3 & 10 & 15 & 10 & 6 \\
\hline Fperciers & & & 2 & 2 & 2 & 3 & 4 & 6 & 5 & 5 & 3 & 3 & 3 & 3 & & 2 & & 26 & 6 \\
\hline Sennes & & & 2 & 2 & 2 & & & & & 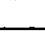 & & t. & 1 & 1 & & & & & 1 \\
\hline \multirow{2}{*}{\multicolumn{3}{|c|}{$\begin{array}{l}\text { Nombre de camets consideres } \\
\text { Proportion de professionnels }\end{array}$}} & 55 & 56 & 73 & 92 & 80 & 159 & 181 & 179 & 170 & 149 & 138 & 113 & 143 & 80 & 35 & 32 & 237 \\
\hline & & & $38 \%$ & $41 \%$ & $33 \%$ & $29 \%$ & $31 \%$ & $19 \%$ & $19 \%$ & $20 \%$ & $20 \%$ & $21 \%$ & $22 x$ & $26 \%$ & $6 \%$ & $8 \%$ & $23 \%$ & $9 \%$ & $11 \%$ \\
\hline
\end{tabular}




\subsection{Répartition spatio-temporelle des types}

Contrairement aux résultats de l'étude quantitative, l'AFC du calendrier (Tableau VI) fait ressortir trois saisons : le premier plan factoriel oppose les mois "froids" (janvier, février, mars), "chauds" (juin, juillet, août) et "intermédiaires" (Fig. 7). Les lignes structurantes sont les pièges de type 1, associés aux saisons intermédiaires, les filets de type 1 et les carrelets de type 1 associés à la saison chaude ainsi que les pièges de type 3 associés à la saison froide.
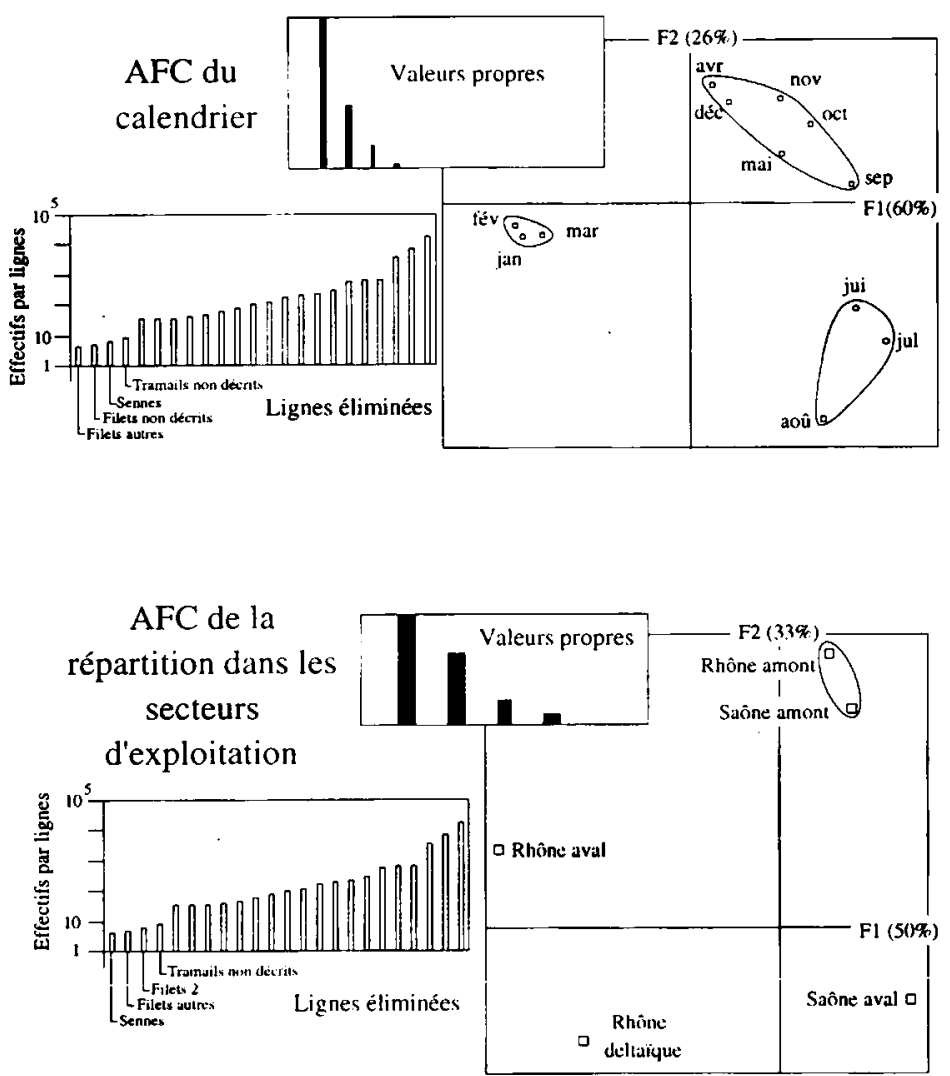

Figure 7 : Plans factoriels F1 x F2 des colonnes de l'AFC du calendrier de pêche (en haut) et de la répartition des engins (en bas) dans les secteurs d'exploitation. Les valeurs propres associées et les lignes éliminées sont précisées.

Figure 7 : F1 $\times$ F2 factorial map of the columns of the Correspondence Analysis for the fishing calendar (top) and the distribution of the fishing gear (bottom) in the exploitation sectors. Eigen values and eliminated rows are given.

L'AFC de la répartition entre secteurs d'exploitation (Tableau VII) rassemble les parties amont des cours d'eau et disperse les autres secteurs aux quatre coins du premier plan (Fig. 7). Les lignes structurantes sont les pièges de type 1 associés au Rhône deltaïque et au Rhône aval, les filets de type 3 sur les parties amont des cours d'eau et les pièges de type 3 sur la Saône aval.

\section{DISCUSSION-CONCLUSION}

\subsection{Une activité de pêche peu développée et en déclin}

Que ce soit en nombre de pêcheurs ou en nombre d'autorisations, qu'elle soit amateur ou professionnelle, la pêche aux engins sur l'ensemble du DPF du Rhône est 
nettement moins importante que celle des migrateurs en Gironde (CASTELNAUD et al., 1985). La distinction entre la pêche amateur et la pêche professionnelle reste primordiale en relation avec les cahiers des charges qui autorisent un nombre d'engins plus important aux professionnels.

BABIN (1991) situe l'activité professionnelle fluviale sur le bassin à un niveau équivalent à celui des lacs alpins avec environ $10 \%$ des entreprises de pêche continentale françaises (marins pêcheurs exclus). Le nombre de pêcheurs professionnels que nous obtenons est légèrement supérieur au dénombrement exhaustif de CASTELNAUD et BABIN (1990) (37 contre 34 après retrait des 7 pêcheurs exerçant sur le Doubs et l'étang de Montaubry). Les recoupements susceptibles d'isoler l'activité d'un même individu ayant rendu plusieurs carnets sont impossibles dans nos données en raison de l'anonymat des réponses, établies conformément aux lois du 7 juin 1951 et du 6 janvier 1978 relatives aux statistiques, à l'informatique et aux libertés.

Le nombre de timbres délivrés en France pour la pêche amateur aux engins se situe aux alentours de 10000 par an (ANONYME, 1992c).Le nombre de pêcheurs amateurs sur le Rhône et la Saône n'en représentent que $7 \%$, alors que le bassin couvre $15 \%$ du territoire national.

On peut être tenté d'interpréter l'intensité de pêche annuelle (en nombre d'autorisations $/ \mathrm{km} / \mathrm{secteur} / \mathrm{an}$ ) comme une densité de pêcheurs par secteur d'exploitation. Si cela est proche de la réalité pour les amateurs, plus d'un professionnel sur deux détient au moins deux autorisations. En effet, ces pêcheurs doivent généralement exploiter plusieurs lots pour assurer leur subsistance. Or les autorisations sont délivrées lot par lot. Toutefois, même si cet indice surévalue le nombre de professionnels, il n'atteint jamais les 3 pêcheurs par kilomètre obtenus sur les cours d'eau navigables en 1902 par CARDAILLAC de St PAUL si on exclut les individus qui, à l'époque, étaient admis sans autorisation. De même, sur le Mississippi, pendant les années 80, FREMLING et al. (1989) rapportent des densités de pêcheurs commerciaux plus importantes (2 100 licences sur $1148 \mathrm{~km}$ dans le Haut Mississippi et 1800 licences sur $1570 \mathrm{~km}$ dans le Bas Mississipi) alors que le nombre de pêcheurs à l'hectare de ce fleuve se classe dans la moyenne des fleuves mondiaux (WELCOMME, 1985).

La distinction de sept familles fait ressortir un appauvrissement des procédés de pêche depuis le début du siècle malgré quelques innovations techniques peu nombreuses (nappes en nylon monofilament, cerf-volant). En effet, DROIN DE BOUVILLE et DAUBREE (1900) répertoriaient sur le même domaine dix familles distinctes et une centaine de noms d'engins différents. Ce phénomène est aussi signalé par BACALBASADOBROVICl (1989) sur le Danube depuis le début des années 70.

En conclusion, la faible densité de pêcheurs et la baisse de la diversité des modes de pêches confirment la déprise des pêcheurs aux engins sur le DPF du bassin du Rhône. Les archives permettent de suivre localement le déclin des effectifs depuis les années trente (SORNAY, 1932 ; KIENER, 1985 ; DDAF de l'Ain, 1988 ; BRAVARD, 1987).

\subsection{Précision et biais de la méthode}

Le nombre de réponses obtenues a été suffisant pour quantifier le nombre d'engins de manière satisfaisante. Près d'un pêcheur sur deux a répondu spontanément. Les pêcheurs ont visiblement accueilli ces nouvelles dispositions favorablement. Toutefois, trois biais importants subsistent dans l'état actuel des données.

D'abord, la quantification de l'effort de pêche en nombre de jours de pêche se heurte au problème des journées sans pêche qui ne sont pas signalées de façon exhaustive dans les carnets. Mais, contrairement aux obsenvations effectuées sur les pêcheurs à la ligne (ANONYME, 1990 ; CRYER et MACLEAN, 1991), il est permis de penser que les "bredouilles" sont négligeables vu l'efficacité plus importante des procédés de pêche utilisés tant par les professionnels que les amateurs. Cela demande néanmoins à être confirmé.

Ensuite, il a été montré aux Etats-Unis que les déclarations spontanées de pêche récréative étaient effectuées préférentiellement par les pêcheurs les plus performants ; ce qui conduit par extrapolation à des surestimations (DEUEL, 1980a ; DEUEL, 1980b). Mais il 
reste à montrer que ceci est vrai en France, d'autant plus que cela est mis en doute par ELIE et al. (1989) dans l'estuaire de la Gironde. Le problème de savoir si l'hypothèse d'un échantillon aléatoire simple est vérifiée reste toujours une des difficultés majeures de la collecte des données halieutiques (LAUREC et al., 1983).

Enfin, la falsification des déclarations est toujours possible car aucun contrôle n'est effectué auprès des pêcheurs. Toutefois, nous avons sensibilisé les associations de pêcheurs en leur précisant qu'il valait mieux ne pas rendre de carnet plutôt que de faire de fausses déclarations. De plus, contrairement à la situation décrite dans l'estuaire de la Gironde, les enjeux commerciaux étant moins importants sur le bassin du Rhône, les pêcheurs qui l'exploitent ont donc moins de raisons de dissimuler leurs captures.

Bien qu'aucune étude récente ne fasse état de la pêche amateur aux engins en France, cette catégorie de pêcheurs est intéressante du point de vue statistique car, contrairement aux professionnels, ils sont nombreux, ils rendent généralement un carnet par pêcheur et par lot, leur taux de réponses suit une allocation proportionnelle à leur effectif par secteur. Ce qui confère aux estimations plus de précision, dans la mesure où chaque secteur d'exploitation est homogène.

On ne peut pas, non plus, négliger les avantages méthodologiques que présentent des pêcheurs professionnels. Leur fréquentation varie moins au cours de l'année et ils utilisent un nombre important d'engins identiques. Malheureusement, leur activité se concentre sur certaines périodes durant lesquelles la pêche est rentable. Or, le découpage mensuel retenu pour la saisie des données ne suit pas toujours ces campagnes.

\subsection{Rythme saisonnier et caractéristiques locales de l'effort de pêche}

L'étude des différentes intensités de pêche sur le DPF du bassin fait ressortir :

(1) une baisse de fréquentation par les professionnels entre les deux années d'exploitation, (2) un secteur à forte intensité de pêche : la Saône aval, (3) deux secteurs à intensité de pêche moyenne : le Rhône amont et le Rhône deltaïque, (4) deux secteurs à faible intensité de pêche : le Rhône aval et la Saône amont, (5) un rythme saisonnier d'activité avec une forte affluence de juin à novembre.

Les engins utilisés conduisent à des saisons légèrement différentes de celles de l'intensité mensuelie. La saison froide (mois de janvier, février, mars) et les saisons intermédiaîres (de septembre à décembre ainsi que les mois d'avril et mai) se caractérisent par l'emploi des pièges, alors qu'en saison chaude (mois de juin, juillet et août) l'effort se diversifie sous l'influence des amateurs avec, entre autres, les carrelets de petites mailles (Tableau VI). Le calendrier ainsi obtenu intègre celui établi par CASTELNAUD et BABIN (1990) pour les professionnels fluviaux seulement.

Les engins utilisés ne sont pas les mêmes suivant les secteurs (Tableau VI). Le Rhône déltaïque et le Rhône aval se distinguent par l'usage du cerf-volant ou gangui, la Saône aval par celui des petites nasses, les parties amont des cours d'eau par celui des filets de tailles et de maillages moyens.

\subsection{Détermination des métiers de pêche}

L'effort de pêche reste qualitativement suffisamment variable pour poser des difficultés de standardisation en vue de la définition de métiers' de pêche. Dans ces conditions, l'isolement de métiers s'établira, dans une première étape, à partir de l'équipement décrit sous la forme de différentes combinaisons des familles d'engins dominantes (Tableau IV), soit :

- 5 métiers amateurs ( $80 \%$ des réponses de cette catégorie de pêcheurs) correspondant respectivement à des équipements constitués de carrelet(s) uniquement, filet(s) uniquement, carrelet(s)+piège(s), piège(s) uniquement, filet(s)+piège(s) ;

- 3 métiers professionnels ( $74 \%$ des réponses de cette catégorie de pêcheurs) correspondant respectivement à des équipements constitués de pièges uniquement, filets uniquement, filets (tramails)+pièges. 
L'étude approfondie des engins montre que, dans une même famille, les catégories de captures varient beaucoup d'un type à l'autre. De plus, les espèces capturées en majorité ne correspondent pas obligatoirement aux espèces cibles. Pour s'affranchir de ce problème, nous proposons de considérer chaque secteur séparément. Ainsi régionalisée, la définition du métier permet d'assurer une certaine homogénéité d'exploitation. Dans un secteur d'exploitation donné, avec une combinaison de familles d'engins donnée, les espèces cibles ont peu de chance de varier d'un pêcheur à l'autre.

\subsection{Perspectives}

Pour améliorer notre définition des différents métiers de pêche et notre connaissance de l'effort de pêche, il serait nécessaire de disposer d'informations complémentaires sur les espèces cibles de chaque pêcheur en fonction du mois et du type d'engin, le mode d'utilisation des engins (pose, traîne, depuis la rive ou en bateau....) et le nombre de journées sans captures.

Néanmoins, il faut rappeler que les carnets de pêche peuvent fournir des informations sur $707 \mathrm{~km}$ de cours d'eau, soit $64 \%$ du domaine total étudié (Tableau II). Ils mettent à la disposition des gestionnaires et des scientifiques les résultats de plus de 5000 journées de pêche par an et rendent compte de l'utilisation de modes de pêche rarement utilisés dans les inventaires scientifiques.

Enfin, les informations fournies par les carnets, dans leur état actuel, sont suffisantes pour donner une estimation acceptable du nombre total d'engins ainsi que pour établir des métiers de pêche. Le regroupement suivant les secteurs d'exploitation et les mois de pêche résume correctement les variations spatio-temporelles en particulier chez les amateurs. L'étude conjointe des deux catégories de pêcheurs complique certainement l'interprétation des résultats mais permet surtout de diversifier les sources d'informations. II reste à savoir si ces données sont utilisables pour effectuer une estimation des prélèvements et de l'abondance des ressources, ce qui fait l'objet de la seconde partie de cet article consacrée à l'analyse des captures.

\section{REMERCIEMENTS}

Nous tenons à remercier ici tous les pêcheurs aux engins et leurs associations, ainsi que le Service de la Navigation Rhône-Saône, la Direction Régionale de l'Environnement Rhône-Alpes et les différents services gestionnaires qui ont participé à l'établissement de ces statistiques. Nous remercions également Franck TORRE qui nous a spontanément apporté son aide dans l'installation et l'exploitation sous forme informatique de ces données ainsi que Jean ALLARDI et Philippe HENSEL pour leurs commentaires sur le manuscrit.

Ce travail fait partie du programme interdisciplinaire de recherches sur l'environnement du CNRS. II a été financé par une bourse de Docteur Ingénieur.

\section{BIBLIOGRÁPHIE}

ANONYME, 1990. La pêche aux lignes sur le Rhône. Schéma de vocation piscicole du fleuve Rhône, Document n 6, DBRMC, mars 1990, 37 p.

ANONYME, 1992a. L'aquaculture, l'enseignement agricole, le savoir vert : le réseau aquacole acteur du développement, Min. de l'Agriculture et de la Forêt, 3ème volume, $52 \mathrm{p}$.

ANONYME, 1992b. Qui sont les pêcheurs? Gazette officielle de la pêche et de l'eau, 29 mai 1992, 1074, 7-14.

ANONYME, 1992c. Les eaux continentales françaises; une richesse à gérer. Actes du colloque des Rencontres halieutiques de Rennes, E.N.S.A.R., 308 p.

ARRIGNON J., 1991. Aménagement piscicole des eaux douces. Technique et documentation, Lavoisier Eds., Paris, 631 p. 
BABIN D., 1991. Résultats de l'enquête expresse 1989 - Estimation de la production et du chiffre d'affaires des pêches professionnelles continentales françaises (Marins pêcheurs exclus). CEMAGREF/Division ALA, Bordeaux, février 1991, 4 p.

BRANDT A. von, 1968. Classification of fishing gear in Kristjonsson H., Modern fishing gear of the world, 274-296, Fishing news Ltd., London.

BRAVARD J.P., 1987. Le Rhône, du Léman à Lyon. La Manufacture, Lyon, 452 p.

BACALBASA-DOBROVICI N., 1989. The Danube river and its fisheries in DODGE D.P. Proceedings of The International Large River Symposium, 455-468, Can. Spec. Publ. Fish. Aquat. Sci. 106.

CARDAILLAC DE ST PAUL Baron del Péré de, 1902. Droit des pêcheurs à être consultés sur les questions relatives à la pêche. Bulletin de la Société d'aquiculture et de pêche, 14, 366-377.

CASTELNAUD G., CREZUELLE D., GUCHAN A. et ROCHARD E., 1985. La pêche des migrateurs en Gironde, 2ème partie : Enquête socio-professionnelle et proposition de gestion. Rapport CEMAGREF/Division ALA, MSHA/Min. Env./ Dép. Gironde, Bordeaux, septembre 1985, $110 \mathrm{p}$.

CASTELNAUD G. et BABIN D., 1990. La pêche professionnelle aux filets et aux engins dans les eaux continentales françaises-Deuxième partie - Les bassins Rhône, Saône, Doubs, Rhin, Somme, Charente, Garonne-Dordogne, Adour et les lacs alpins. Rapport CEMAGREF/Division ALA, Bordeaux, décembre 1990, $141 \mathrm{p}+$ annexes.

CASTELNAUD G. et BABIN D., 1992. La pêche professionnelle fluviale et lacustre en France. Enquête au fil de l'eau. Etudes Ressources en eau $n^{\circ} 5$, Min. de l'Environnement, CEMAGREF/Division ALA, Bordeaux, $291 \mathrm{p}$.

CECPI , 1978. La valeur et les limites des diverses méthodes de surveillance biologique de la qualite des eaux pour les poissons d'eau douce. Document Technique du CECPI $n^{\circ}$ 32, FAO Publ., Rome, $24 \mathrm{p}+$ figures.

CERESTA, 1986. Aide-mémoire pratique des techniques statistiques. Revue de statistique appliquée, 34(numéro spécial), $274 \mathrm{p}$.

CHESSEL D. et DOLEDEC S., 1991. ADE Software, version 3.1. URA CNRS 1541, Université Lyon I.

COTTEREAU C., 1988. Leçons tirées de l'aménagement du Rhône sur l'hydrobiologie, la qualité de l'eau, et le débit solide. Actes du 16ème congrès de la Commission Internationale des Grands Barrages, 53-83, San Francisco.

CRYER M. et MACLEAN G.D., 1991. Catch for effort in a New Zealand recreational trout fishery - a model and implication for survey design in COWX I.G. Catch effort sampling strategies, 61-71, Fishing news books, Oxford (U.K.).

CTGREF, 1979. Etude halieutique de l'estuaire de la Gironde. Rapport CEMAGREF/Division ALA, Bordeaux, contrat EDF, $189 \mathrm{p}$.

DDAF de l'Ain, 1988. Nombre de licences de pêche aux engins et aux filets entre 1967 et 1988. Archives de la DDAF de l'Ain, Bourg-en-Bresse.

DEUEL D.G., 1980a. Special surveys related to data needs for recreational fisheries in GROVER J.H., Allocation of fishery resources, Proc. of the Technical Consultation on Allocation of Fishery Resources held in Vichy (France), 77-81, FAO Publ..

DEUEL D.G., 1980b. Survey methods used in the United States Marine Recreational Fishery Statistics Program in GROVER J.H., Allocation of fishery resources, Proc. of the Technical Consultation on Allocation of Fishery Resources held in Vichy (France), 82-86, FAO Publ..

DILL W.A., 1990. Inland fisheries of Europe. EIFAC Technical Paper n52. FAO Publ., Rome, $471 \mathrm{p}$. 
DROIN DE BOUVILLE R. de et DAUBREE M.L., 1900. Pêche fluviale en France. Principaux engins et modes de pêche autorisés ou interdits. Ministère de l'agriculture des eaux et des forêts, Imprimerie nationale, Paris, $652 \mathrm{p}$.

ELIE P., VERDILHAC P. de, CASTELNAUD G. et GADRAT S., 1983. Etude de surveillance halieutique de l'estuaire de la Gironde-1981. Rapport CEMAGREF/Division ALA, Bordeaux, juin 1983, $78 p$.

ELIE P., ROCHARD E. et BOIGONTIER B., 1989. Etude de suivi halieutique de l'estuaire de la Gironde-1988. Rapport CEMAGREF/Division ALA, Bordeaux, juin 1989, $169 \mathrm{p}+$ bibliographie.

FAO, 1992. Catches and landings, 1990. FAO Yearbook of fishery statistics, 70, $647 \mathrm{p}$.

FREMLING C.R., RASMUSSEN J.L., SPARKS R.E., COBB S.P., BRYAN C.F. et CALFLIN T.O., 1989. Mississippi river Fisheries : a case history in DODGE D.P. Proceedings of The International Large River Symposium, 309-351, Can. Spec. Publ. Fish. Aquat. Sci. 106.

FRUGET J.F, 1989. L'aménagement du Bas-Rhône. Evolution du fleuve et influence sur les peuplements de macroinvertébrés benthiques. Thèse de Doctorat, Univ. Lyon I, $439 p$

GABIGNON Y., CASTELNAUD G. et ELIE P., 1984. Etude de surveillance halieutique de l'estuaire de la Gironde-1983. Rapport CEMAGREF/Division ALA, Bordeaux, juin $1984,115 p+$ annexes et bibliographie.

GALLOIS G., 1947. Aménagement du Rhône Vivarais par la Compagnie Nationale du Rhône, ses incidences sur l'économie piscicole, mesures tendant à les atténuer. Bull. fr. pisc., 146, 25-34.

GERDEAUX D., 1988. Synthèse des connaissances actuelles sur le peuplement piscicole du lac d'Annecy - Bilan piscicole et halieutique. Rapport INRA, Station d'hydrobiologie lacustre de Thonon-les-bains, octobre 1988, 43 p.

GERDEAUX D., 1990. Fisheries management in an international lake : Lake Geneva in VAN DENSEN W.L.T., STEINMETZ B. et HUGHES R.H., Management of freshwater fisheries, 168-181, Pudoc, Wageningen.

KIENER A., 1985. Au fil de l'eau en pays méditerranéen. Aubanel, Avignon, 308 p.

LAUREC A. et LE GUEN J.C., 1981. Dynamique des populations marines exploitées, tome I: concepts et modèles. Rapport scientifiques et techniques $n^{\circ} 48$, C.N.E.X.O, Brest, novembre 1982, $118 \mathrm{p}$.

LAUREC A., LE GUEN J.C. et FRONTIER S., 1983. Collecte de l'information pour une gestion rationnelle des stocks halieutiques in FRONTIER S., Stratégie d'échantillonnage en écologie, 385-415, Masson, Paris.

MALAVOI J.R., 1989. Typologie des faciès d'écoulement en unité morphodynamiques des cours d'eau à haute énergie. Bull. Fr. Pêche Piscic., 315, 189-210.

MONOD T., 1973. Contribution à l'établissement d'une classification fonctionnelle des engins de pêche. Bull. Mus. natn. Hist. nat.,156 (3ème sér.), Ecologie générale 12, 205-231.

PERAU M.A., 1930. La statistique des pêche fluviales. Bull. fr. pisc., 26, 29-30.

PERSAT H., 1988. De la biologie des populations de l'Ombre commun Thymallus thymallus (L. 1758) à la dynamique des communautés dans un hydrosystème fluvial aménagé, le Haut-Rhône français. Eléments pour un changement d'échelle. Thèse d'état, Univ. Lyon I, $223 \mathrm{p}$.

SAPORTA G., 1990. Probabilités, analyse des données et statistiques. Tecnip, Paris, 493 p.

SCHERRER B., 1983. Techniques de sondage en écologie in FRONTIER S., Stratégie d'échantillonnage en écologie, 65-162, Masson, Paris.

SORNAY, 1932. La consommation du poisson d'eau douce à Lyon. Bull. Fr. Piscic., 54, 180-185. 
Bull. Fr. Pêche Piscic. (1993) 330 : 245-269 - $269-$

WELCOMME R.L., 1978. Some aspects of the use of inland waters for recreational fisheries in ALBASTER J.S., Recreational freshwater fisheries : their conservation, management and development, 23-34, Water Research Center Publ., Medmenham, U.K.

WELCOMME R.L., 1985. River fisheries. FAO Fish. Tech. Paper, 262, 330 p. 